\title{
1 ULK4 and Fused/STK36 interact to mediate assembly of a motile
}

2 flagellum

3

4 Running title

$5 \quad$ ULK4 and Fused/STK36 in motile cilia

6

\section{Authors}

8 Ciaran J. McCoy ${ }^{1,4}$, Humbeline Paupelin-Vaucelle ${ }^{1}$, Peter Gorilak ${ }^{2}$, Tom Beneke ${ }^{1}$, Vladimir 9 Varga $^{2}$ and Eva Gluenz ${ }^{1,3, *}$

\section{Affiliations}

$12{ }^{1}$ Sir William Dunn School of Pathology, University of Oxford, Oxford, United Kingdom

132 Institute of Molecular Genetics of the Czech Academy of Sciences, Prague, Czech 14 Republic

$15{ }^{3}$ current affiliation: Wellcome Centre for Integrative Parasitology, Institute of Infection, 16 Immunity \& Inflammation, College of Medical Veterinary and Life Sciences, University of 17 Glasgow, Glasgow, United Kingdom

$18{ }^{4}$ current affiliation: Microbes \& Pathogen Biology, The Institute for Global Food Security, 19 School of Biological Sciences, Queen's University Belfast, Belfast, United Kingdom

*Corresponding author: eva.gluenz@glasgow.ac.uk

22

\section{Keywords}

24 ULK4, Fused/STK36, flagella, Leishmania, ependymal cilia 


\section{Summary Statement}

27 Knockout phenotypes in Leishmania, and confirmation of ULK4 ciliary localisation in mouse,

28 show ULK4 and Fused/STK36 interact in a conserved pathway for stable assembly of motile 29 cilia.

\section{Abstract}

31 Unc-51-like kinase (ULK) family serine-threonine protein kinase homologs have been linked 32 to the function of motile cilia in diverse species. Mutations in Fused/STK36 and ULK4 in mice 33 resulted in hydrocephalus and other phenotypes consistent with ciliary defects. How either 34 protein contributes to the assembly and function of motile cilia is not well understood. Here 35 we studied the phenotypes of ULK4 and Fused gene knockout (KO) mutants in the 36 flagellated protist Leishmania mexicana. Both KO mutants exhibited a variety of structural 37 defects of the flagellum cytoskeleton. Biochemical approaches indicate spatial proximity of 38 these proteins and indicates a direct interaction between the N-terminus of LmxULK4 and 39 LmxFused. Both proteins display a dispersed localisation throughout the cell body and 40 flagellum, with enrichment near the flagellar base and tip. Fused/STK36 was previously 41 shown to localise to mammalian motile cilia and we demonstrate here that ULK4 also 42 localises to the motile cilia in mouse ependymal cells. Taken together these data suggest a 43 model where the pseudokinase ULK4 is a positive regulator of the kinase Fused/STK36 in a 44 pathway required for stable assembly of motile cilia. 
46

47

48

49

50

51

52

53

54

55

56

57

58

59

60

61

62

63

64

65

66

67

68

69

70

71

72

73

74

75

76

77

78

79

80

81

\section{Introduction}

Serine-threonine protein kinases of the Unc-51-like kinase (ULK) family are found across eukaryotic species, with the best-characterised members found in metazoans and plants. In mammals the family has four active kinases (ULK1, ULK2, ULK3 and Fused/STK36) and one pseudokinase ULK4. The active kinases function in multiple well-studied biological processes: ULK1 and ULK2 act in autophagy pathways in starvations responses (Lee, 2011), ULK3 is a regulator of the sonic hedgehog signalling pathway (Maloverjan et al., 2010) and Fused kinase was first identified in Drosophila (Preat et al., 1990) as a kinase of Hedgehog $(\mathrm{Hh})$ signalling pathway. Unexpectedly, studies on the mouse Fused homolog, also named Serine/threonine-protein kinase 36 (STK36), showed that fused knockout mice did not show any defects in Hh signalling (Chen et al., 2005; Merchant et al., 2005). Instead, it was found that these mice developed hydrocephalus and respiratory infections and died a few weeks after birth (Merchant et al., 2005). This phenotype, together with the finding that Fused was highly expressed in ependymal cells and nasal epithelium, pointed to a function in cells with motile cilia.

Cilia and flagella are cellular appendages formed by a microtubule axoneme, which originates at the basal body and has more or less extensive structural elaborations, depending on its specific function within a cell type. A key distinction is between motile cilia/flagella, which typically have a so-called 9+2 axoneme composed of a pair of singlet microtubules (central pair; CP) surrounded by a ring of nine doublet microtubules. The latter are decorated with axonemal dyneins and other protein complexes such as radial spokes (RSP) required for bending. They provide one of the main modes of eukaryotic cell motility, as used by many protists, unicellular plants and gametes of diverse species including mammalian sperm cells. Cells extending motile cilia are also found within tissues where they generate directional fluid flow required for development and normal physiology (Ringers et al., 2020), e.g. ciliated ependymal cells of the brain ventricles and spinal canal help the circulation of cerebrospinal fluid. Primary cilia by contrast have a simpler axoneme structure consisting of 9 doublet microtubules and they transduce chemical and mechanical signals from the environment (Goetz and Anderson, 2010). Cilia and flagella construction relies on a conserved intraflagellar transport (IFT) system that shuttles cargo, including axonemal constituents, from the basal body to the axoneme tip and back (Lechtreck, 2015).

Wilson et al. (2009) Investigated whether mammalian Fused was required for the $\mathrm{Hh}$ responses mediated through primary cilia and found that this was not the case: $\mathrm{Fu}^{-/}$mice were capable of constructing normal primary cilia. By contrast, $\mathrm{Fu}^{-/-}$mice had motile cilia defects with $60 \%$ showing an abnormal ultrastructure ( $40 \%$ of cilia had no central pair) and misalignment, which resulted in uncoordinated ciliary beating. Unlike the situation in mice, 
82

83

84

85

86

87

88

89

90

91

92

93

94

95

96

97

98

99

100

101

102

103

104

105

106

107

108

109

110

111

112

113

114

115

116

117

morpholino-mediated knockdown of $F u$ in Zebrafish did affect Hh signalling, and in addition, the morphants also had structurally and functionally disrupted cilia in Kupffer's vesicle, which are required in development to generate a directional fluid flow to establish the body's leftright axis. The effect on the cilia was independent of $\mathrm{Hh}$ signalling, indicating that this kinase acts in multiple conserved pathways in different lineages (Wilson et al., 2009). Studies on oviduct cilia provided further evidence for an important role of mammalian Fused/STK36 in motile cilia. The majority of oviduct cilia of $\mathrm{Fu}^{-/-}$mice had an abnormal axoneme structure (Nozawa et al., 2013): cilia were misaligned and a majority of these oviduct cilia lacked central pair microtubules, within tissues that also produced some cilia with a normal 9+2 architecture. Mouse sperm flagella function were also affected by conditional deletion of $F u$, showing motility defects associated with infertility (Nozawa et al., 2014). In the sperm cells the 9+2 microtubule structure of the axoneme was maintained but they exhibited a defect in the structure of the manchette, a transient microtubule structure required for correct shaping of the sperm head.

In humans, primary ciliary dyskinesia (PCD) is a disease caused by defects in motile cilia, which can result from varied genetic causes. Fused/STK36 loss-of-function mutations were found in a PCD patient (Edelbusch et al., 2017) where most motile cilia were normal but $~ 5 \%$ had abnormal microtubule numbers and cilia mis-orientation affected their movement. The Fu protein itself was localised along the length of the motile cilium (Edelbusch et al., 2017; Nozawa et al., 2013), consistent with the genetic evidence for a role of Fused/STK36 in motile cilia. One study suggested it was sited between the radial spoke heads and the central pair microtubules (Edelbusch et al., 2017). Biochemical evidence for an interaction between mouse Fu and the central pair-associated protein Spag16/PF20 and several other ciliary proteins led to a model where Fu and the kinesin-like protein Kif27 interact to promote CP assembly in motile cilia of mammals (Wilson et al., 2009).

Less functional information is available for the pseudokinase UKL4, but, like for Fused/STK36, there is converging evidence for a function associated with motile cilia. In a mouse knockout and phenotyping screen, ULK4 was identified in a cohort of genes for which disruption caused hydrocephalus (Vogel et al., 2012). This gene cohort also included Fused/STK36 and Kif27. In Ulk4 hypomorph mutant mice, in which Ulk4 expression was reduced, ependymal cells had abnormal axonemes and disorganised cilia (Liu et al., 2016). Abnormal structures included $9+0$ and other deviations from normal $9+2$ microtubule numbers, while some cilia had normal structures. The possibility was raised that UKL4 regulates different processes of ciliogenesis. RNA-seq showed alterations in cilia related transcripts and indicated that UKL4 regulated Foxj1 transcription factor pathways involved in ciliogenesis. 
118 The documented links between ULK4 disruption and human diseases prompted the 119 elucidation of the ULK4-ATPYS complex structure and mapping of the molecular 120 'environment' by proximity labelling (Preuss et al., 2020). The proximity interaction network 121 uncovered five kinases, including Fused/STK36, and proteins associated with a range of 122 different functions, amongst them ciliary and microtubule-associated proteins.

123 Orthologs of Fused/STK36 and UKL4 are present in the kinetoplastids, a group of flagellated protozoans that are well-studied on grounds of their medical importance as causative agents of neglected parasitic diseases. In Trypanosoma brucei, FCP6/TbFused and FCP5/TbULK4 localised to the flagella connector (Varga et al., 2017), a mobile transmembrane structure at the tip of the newly growing flagellum that connects it to the existing flagellum during a defined phase in the growth cycle (Moreira-Leite et al., 2001). Deletion of LmxFused and LmxULK4 in Leishmania mexicana, in a 'kinome'-wide knockout (KO) screen yielded cells with shorter flagella and reduced motility (Baker et al., 2021). We independently found LmxUKL4 and LmxFused in a screen of flagellar protein $\mathrm{KO}$ mutants with reduced motility. Here, we conducted a detailed analysis of these null mutant phenotypes and found that LmxULK4 and LmxFused KOs both present with the same spectrum of morphological defects, affecting length and ultrastructure of the flagellar axoneme. This indicates a conserved role for Fused/STK36 or ULK4 in ciliogenesis and cilia function. Our data support a model whereby these two proteins directly interact with each other to promote stable assembly of motile flagella in evolutionarily distant lineages. Consistent with this model, we also show that the mammalian ULK4 protein localises to the motile cilium of mouse ependymal cells.

\section{Results}

141 LmxULK4 and LmxFused localise to both the Leishmania cell body and motile 142 flagellum

143 LmxULK4 and LmxFused encode UNC-51-like proteins that are orthologs of animal ULK4 144 and Fused/STK36, respectively (Fig 1A). Like its mammalian orthologs, LmxULK4 represents 145 a catalytically inactive pseudokinase, as supported by the lack of the catalytically important

146 Lysine residue within the typically highly conserved 'VAIK' motif (see Fig 1B). We assessed 147 protein localisation by inserting an mNeonGreen (mNG) fusion tag at the endogenous gene 148 locus (tagging both alleles of the gene) and imaging the fusion proteins in live cells. Both 149 proteins localised to the Leishmania promastigote cell body and motile flagellum throughout 150 the cell cycle (Fig 1C-E). Individual cells (both dividing and non-dividing) often displayed 151 enriched signal at the flagellum tip, base or both but no evidence was found for a dominant 152 signal uniquely localised at the tip of the newly growing flagellum, where the orthologous $T$. 153 brucei proteins are localised (Varga et al., 2017). The LmxFused::mNG and LmxULK4::mNG 
154 fluorescent signal was absent in cells extracted with a nonionic detergent (S1 Fig),

155 suggesting that neither protein is tightly attached to the insoluble cytoskeleton.

156 Loss of LmxULK4 or LmxFused leads to failure of normal motile flagellum assembly

157 To analyse loss-of-function phenotypes, CRISPR/Cas9 was used to knock out (KO)

158 LmxULK4 and LmxFused, respectively (S2 Fig). Measurement of swimming speed showed

159 that this was significantly reduced in both $\mathrm{KO}$ lines (Fig 2A), relative to parental controls,

160 consistent with a lack of a long flagellum in the majority ( 90-95\%) of cells in these KO

161 populations $(n \geq 500$; Fig $2 B, D)$. The visible flagella that were still present in a minority of the

$162 \mathrm{KO}$ cells were shorter than those of the parental controls ( $\mathrm{n} \geq 27$; Fig 2C; one-way ANOVA

163 with the Šídák correction for multiple comparisons). Moreover, although these were not

164 completely paralysed, they did not enable cell propulsion but rather facilitated a small

165 proportion of cells to twitch at the bottom of their culture dish when observed in vitro. Despite

166 these striking changes in morphology, the KO mutants only exhibited a very slight increase in

167 doubling time (S3 Fig). Importantly, the flagellum-associated phenotypes reported here were

168 completely rescued via re-introduction of the target gene on an episomal addback plasmid

169 for the $\Delta L m x$ Fused cell line and partially rescued for $\Delta L m x U L K 4$ (Fig $2 \mathrm{~A}-\mathrm{C}$ ). A dual $K O$ of

170 both genes ( $\triangle L m x U L K 4 / \Delta L m x$ Fused) resulted in flagellar phenotypes that were

171 indistinguishable to either individual $\Delta L m x U L K 4$ and $\Delta L m x F u s e d$ cell line (Fig 2A-D).

$172 \Delta L m x U L K 4$ and $\Delta L m x F u s e d$ mutants display a range of structural defects in the

173 flagellum

174 These data are consistent with a role of $L m x$ Fused and $L m x U L K 4$ in the assembly of a motile 175 flagellum (Baker et al., 2021). A striking feature of the KO phenotypes was that within clonal 176 populations of apparently aflagellate cells, a small but stable proportion of cells did still 177 possess a long flagellum. This suggests that LmxULK4 or LmxFused are not strictly essential 178 for the formation of a flagellum, but their absence prevented the reliable assembly of 179 functional motile flagella in most cells. To understand which step of flagellum biogenesis 180 failed in these mutants, the structure of the flagella in the $\Delta L m x F u s e d$ and $\Delta L m x U L K 4$ 181 populations was examined in detail. The axonemal radial spoke protein 11 (RSP11; 182 LmxM.09.1530) and the central pair associated protein PF16 (LmxM.20.1400) were chosen 183 as reporter proteins for key structures of the motile axoneme and a cell line was generated 184 simultaneously expressing RSP11::eYFP and PF16::mStrawberry (Pf16::mStr) fluorescent 185 fusion proteins. In this cell line, $L m x F u s e d$ and $L m x U L K 4$ were then deleted, respectively 186 (Fig $3 \mathrm{~A}-\mathrm{B}$ ). This showed that $\Delta L m x$ Fused and $\Delta L m x U L K 4$ exhibit highly similar proportions of 187 multiple distinct flagellar morphotypes and effectively phenocopy each other (Fig 3B). 2/3 of 188 cells in both $\mathrm{KO}$ cell lines not only lacked a long external flagellum (as observed in the 189 untagged cell lines (Fig 2B, D), but the absence of RSP11::eYFP and PF16::mStr fluorescent 
190 signals at the anterior cell pole, where the flagellum normally emerges from the flagellar pocket, indicated that these cells lacked axonemes entirely (Fig 3B, v).

192 Defects in the CP were previously reported for distinct ciliated tissues in both ULK4 and 193 Fused/STK36 mutant mice (Liu et al., 2016; Nozawa et al., 2013; Wilson et al., 2009). 194 Moreover, the central pair microtubule associated protein PF20 (but not PF16) has previously been shown to co-immunoprecipitate with Fused/STK36 when expressed in HEK 293T cells (Wilson et al., 2009). Examination of PF16::mStr signals (Fig 3A-B) found that a majority of cells with a long flagellum still possessed a continuous PF16 signal (Fig 3B), suggesting that the CP was still assembled in these cells, and a disrupted signal was only observed in a small percentage of flagella. To examine whether PF20 was affected by the loss of $L m x F u s e d$ and $L m x U L K 4$, each gene was deleted in a reporter cell line expressing PF16::eYFP / mStr::PF20 (Fig 3C-D). Focusing on the external flagella that still showed a continuous PF16::eYFP signal, we measured the intensity of fluorescence and detected a reduced signal intensity for mStr::PF20 in both $\Delta L m x F u s e d$ and $\triangle L m x U L K 4$ cell lines, while no reduction of the PF16::eYFP signal was seen (Fig. 3E-F). This suggests that PF20 appears to be preferentially lost from $\triangle L m x F u s e d$ and $\triangle L m x U L K 4$ axonemes. In the reciprocal experiments, KO of LmxPF20 did not appear to impact either LmxULK4::mNG or LmxFused::mNG expression or localisation (S4 Fig). Consistent with these findings, cross-sections through free flagella imaged by transmission electron microscopy (TEM) showed normal 9+2 microtubule arrangements and PFR structures in the majority of flagella (Fig 4A, i-iii). No obvious central pair defects were noted in any of these TEM profiles, consistent with the observed RSP11::eYFP and PF16::mStr

212 signals in the majority of external flagella and despite the reduction of PF20 signal (Fig 3).

213 Since fewer than $1 \%$ of cells in both $\Delta L m x$ Fused and $\Delta L m x U L K 4$ populations showed a non-

214 continuous or absent PF16::mStr signal (Fig 3B), the number of external flagella assessed by 215 TEM was likely too small to capture those rare cells. We also noted that one out of the 216 thirteen $\triangle L m x F$ used flagella captured by TEM displayed a $10+2$ microtubule arrangement 217 (Fig 4A, iv), indicating that a variety of ultrastructural defects may be present within sub-sets 218 of these long external flagella.

219 Since fluorescence microscopy data showed that $\sim 25 \%$ of KO cells possessed a short 220 flagellum that failed to extend significantly beyond the neck region of the flagellar pocket (Fig. 3 , iii-iv), the structure of axonemes inside the flagellar pocket was examined next. Here, only $38 \% \Delta L m x F u s e d$ and $27 \%$ of $\triangle L m x U L K 4$ axonemes viewed by TEM showed a $9+2 \mathrm{MT}$ arrangement, compared to $100 \%$ in the parental controls (Fig 4B, C). A variety of defects were noted in both central pair and outer microtubule doublet number and orientation (Fig 4B, C), including mis-positioned outer doublet MT (Fig 4B, iii), <9 doublet MTs (Fig 4B, v and 
227 lacking a CP (Fig 4C) was consistent with the absence of a PF16::mStr signal in about half of 228 the short axonemes viewed by fluorescence microscopy (Fig 3B). To understand better 229 where the defects originated, serial TEM sections spanning the basal-body, transition zone 230 and axoneme regions were examined (S5 Fig A-E). Some cells only extended a few singlet 231 or doublet microtubules from a basal body (S5 Fig B,C); such cells were likely scored as 232 lacking axonemes entirely in Fig 3 (category $v$ in Fig 3B). Other cells had more subtle 233 defects, showing alteration of MT numbers or positioning (S5 Fig D,E). Taken together this 234 suggests that all MT structures of the flagellum are sensitive to the loss of LmxFused and $235 L m x U L K 4$ but none are strictly dependent on these proteins for their formation. IFT recruitment and processivity correlates with $\Delta L m x F u s e d$ and $\Delta L m x U L K 4$ flagellar morphotype

238 To determine whether the loss of $L m x F u s e d$ and $L m x U L K 4$ affected intraflagellar transport, 239 two cell lines were generated that expressed fluorescently tagged IFT proteins, 240 mNG::IFT81/LmxM.33.0230 and mNG::IFT140/LmxM.31.0310 (components of the IFT-B and 241 IFT-A subcomplexes, respectively (Nakayama and Katoh, 2018)). LmxFused and LmxULK4 242 were then knocked out in these reporter lines, which presented with the typical flagellar 243 abnormalities described above. In the minority population that still possessed a long 244 flagellum, IFT particle numbers and velocity were measured, and the results showed that the 245 trains migrated at a relatively normal velocity along $\Delta L m x F u s e d$ and $\Delta L m x U L K 4$ flagella (S6 246 Fig A-N). This analysis demonstrates that in the absence of LmxFused and LmxULK4 at 247 least some cells retain the capacity for normal IFT in fully grown flagella and does not 248 preclude the possibility that loss of $L m x F u s e d$ or $L m x U L K 4$ KO could directly impede IFT in 249 newly growing flagella or in the cells that fail to construct an axoneme at all. Indeed 250 examination of the fluorescence signals showed that a minority population of $\Delta L m x U L K 4$ and $251 \Delta L m x$ Fused cells didn't have IFT signal foci in the basal body region. The mNG::IFT81 signal 252 was absent in $6 \%$ of $\Delta L m x$ Fused cells and in $7 \%$ of $\triangle L m x U L K 4$ cells; the mNG::IFT140 253 focus at the flagellum base was absent in $37 \%$ of $\Delta L m x F u s e d$ cells and in $28 \%$ of $254 \Delta L m x U L K 4$ cells (S6 Fig O,P). Taken together these data show that the IFT behaviour in $255 \Delta L m x$ Fused and $\Delta L m x U L K 4$ cells correlated with the flagellar morphotype. It remains to be 256 tested whether this apparent reduction in IFT protein recruitment is caused by the loss of 257 LmxFused or LmxULK4, and thus a primary cause of flagellum assembly failure, or whether 258 reduced IFT recruitment is an indirect downstream consequence of structural defects 259 resulting from $L m x F u s e d$ and $L m x U L K 4$ KO loss. 
$\Delta L m x U L K 4$ mutants display reduced a-tubulin acetylation, though $\Delta L m x F u s e d$ mutants do not

262 A recent study has shown that RNAi mediated knockdown of ULK4 resulted in reduced levels of acetylated a-tubulin in both primary cultured mouse neurons and brain sections (Lang et al., 2016), though that study did not examine cilia or flagella directly. Alpha-tubulin acetylation has previously been linked to increased microtubule stability and flexibility (see Janke and Montagnac, 2017; Portran et al., 2017). To test the a-tubulin acetylation levels in the L. mexicana parental and KO cell lines, cells were stained with antibody C3B9. This revealed a strong signal along the length of the flagellum and a cortical cell body signal, indicating detection of acetylated tubulin in the axonemal microtubules as well as the cortical cytoskeleton (Fig 5A). Knockout of LmxULK4 resulted in a reduction in acetylated a-tubulin signal in both the Leishmania cell body and flagellum (Fig 5A-B). That signal was restored upon expression of an episomal add-back copy of LmxULK4 (Fig 5A-B). However, no such reduction in acetylated a-tubulin was noted in the $\Delta L m x$ Fused cell line (Fig 5A-B). As a control, we knocked out LmxaTAT1 (LmxM.25.1150), the ortholog of the dominant mammalian a-tubulin acetyltransferase. These cells showed a near complete loss of acetylated a-tubulin signal (Fig 5A-B). Interestingly, however these cells possessed normal long flagella (Fig 5C), and swam normally when observed in culture. Taken together, these data show that the level of a-tubulin acetylation did not correlate with the capacity to build normal motile flagella in these Leishmania mutants, and that the flagellar defects seen in $\triangle L m x F u s e d$ and $\triangle L m x U L K 4$ cell lines were not caused by a reduction in tubulin acetylation. ULK4 may instead regulate a-tubulin acetylation in a Fused/STK36 independent manner in Leishmania, and perhaps mammals as well (Lang et al., 2016).

284 The similarities of the $\Delta L m x F u s e d$ and $\Delta L m x U L K 4$ flagellar phenotypes individually and in combination (see Fig 2-4) suggests that LmxFused and LmxULK4 may act in a common pathway. To determine whether they are in close spatial proximity, and to identify other proximal proteins or complexes, we tagged both alleles of LmxFused with the promiscuous E. coli-derived biotin ligase BirA $^{*}$ and preformed proximity dependent biotinylated protein identification (BiolD (Roux et al., 2012)). Expression of LmxFused::BirA* had no effect on the growth of the cells in culture (S7 Fig A) and the protein showed the expected localisation (S7

291 Fig B). Biotinylated proteins were captured with streptavidin-coupled beads and western blot 292 analysis confirmed the presence of the bait protein LmxFused::BirA* in the eluted fraction 293 (S7 Fig C), which was then analysed by nano-LC/MS/MS. The parental cell line subjected to 294 the same pull-down and MS analysis served as a control. As expected, several 295 endogenously biotinylated proteins (putative carboxylase subunits) were identified and highly 
296 represented in both samples (supplemental data file 1). We used SINQ analysis (Trudgian et

297 al., 2011) to test for enrichment of biotinylated proteins in the LmxFused::BirA* line and 298 identified 125 enriched proteins (supplemental data file 1): 47 were only detected in the 299 LmxFused::BirA* sample (supplemental data file 1 and Fig $6 \mathrm{~A}$ ) and 78 proteins showed $\geq 2$ 300 fold enrichment within the LmxFused::BirA* sample (supplemental data file 1 and Fig 6B).

301 Notably, LmxULK4 was the most highly represented protein identified specifically within the 302 LmxFused::BirA* sample after the bait protein itself (Fig $6 A)$, suggesting that these proteins 303 are in close proximity to each other in the cell. To test whether there was direct physical 304 interaction between LmxFused and LmxULK4, we performed immunoprecipitation, in a cell 305 line expressing LmxFused::eYFP and LmxULK4::MYC. Expression of fusion proteins was 306 confirmed by fluorescence imaging (LmxFused::eYFP, S8 Fig A) and western blots of whole 307 cell lysates (both proteins, S8 Fig B and C). Cell lysates were incubated with anti-GFP 308 conjugated beads and protein pulldown of LmxFused::eYFP was confirmed by western blot 309 (S8 Fig B). A control cell line expressing GFP served as a control for the pull-down and MS 310 analyses (S Fig 8 D). The eluates were analysed by mass spectrometry (supplemental data 311 file 2). The two highest scoring hits were a carbamoyl-phosphate synthase (LmxM.16.0590) 312 and LmxULK4. Notably, 29 of 30 ULK4 peptides mapped specifically to the N-terminus of 313 LmxULK4 (S8 Fig E), within the first 340 out of 1315 amino acids. Western blots probing for 314 the C-terminal tag on LmxULK4::MYC suggested the protein was cleaved or degraded 315 following cell lysis (S8 Fig C). The detection of peptides derived from the LmxULK4 N316 terminus in IP eluates is consistent with a direct physical interaction between LmxFused and $317 \mathrm{~N}$-terminal region of LmxULK4, which also agrees with data on mammalian ULK4 interacting 318 via its kinase domain with Fused/STK36 (Preuss et al., 2020).

319 Given the data presented above supporting an interaction between LmxFused and 320 LmxULK4, we investigated the impact of gene KOs for one of these proteins on the 321 subcellular localisation of the other protein. LmxULK4 KO did not appear to affect LmxFused::mNG localisation (Fig 6D). In the equivalent reciprocal experiment, the signal from LmxULK4::mNG (both alleles tagged) disappeared upon deletion of LmxFused, suggesting that the stable expression of LmxULK4 was dependent on the presence of LmxFused (Fig 6E). These data further corroborate the KO phenocopy and together with the LmxFused BiolD and Co-IP results, which both identified LmxULK4 as high-scoring interaction partner, support the hypothesis that LmxULK4 and LmxFused physically and functionally interact to contribute to the assembly of a normal motile eukaryotic flagellum. Interactions with other flagellar proteins

330 The targets of the LmxFused kinase remain unknown. BiolD and Co-IP both identified 331 additional proteins, albeit with little overlap between the two methods (supplemental data 
332 files 1 and 2). Of the 24 proteins identified in the co-immunoprecipitation with

333 LmxFused::eYFP ( $\geq 3$ spectral counts), 15 were also co-immunoprecipitated with the soluble

334 GFP pulldown control sample, suggesting that these proteins directly interact with eYFP tag

335 as opposed to LmxFused itself (supplemental data file 2). Besides LmxULK4, there were

336 only six proteins detected with $>2$ peptides in the LmxFused Co-IP sample (and $<2$ peptides

337 in the GFP pulldown and parental control samples): LmxM.16.0590 (putative carbamoyl-

338 phosphate synthase), LmxM.11.0940 and LmxM.26.1450 (both hypothetical proteins, present

339 in the flagellar proteome (Beneke et al., 2019)), LmxM.36.4330 (the L. mexicana orthologue

340 of $T$. brucei flagellar attachment zone (FAZ) protein FAZ22 (Zhou et al., 2018)),

341 LmxM.08_29.2510 (glycosomal i-dependent 6-phosphofructokinase) and LmxM.30.1910

342 (hypothetical protein) (Fig 6C). Further experiments will be required to establish which, if any,

343 of these are genuine interaction partners of LmxFused/LmxULK4.

344 In addition to identifying components of a protein complex, BiolD could provide information

345 about the subcellular domains where LmxFused resides, identifying proteins that do not

346 directly interact with LmxFused but that are in close proximity to it. Besides LmxULK4, the

347124 enriched proteins included 23 that have been previously identified within the L. mexicana

348 flagellar proteome (Beneke et al., 2019) and 7 that associate with the FAZ, consistent with

349 the observed subcellular localisation pattern of LmxFused. We hypothesised that potential

350 substrates of LmxFused, or direct interaction partners may be found amongst the most highly

351 enriched proteins in the LmxFused::BirA* sample. We used gene KO to determine whether

352 deletion of these genes had a similar effect as loss of Fused, and whether a dual KO

353 modulated the $\Delta L m x F u s e d$ phenotype, as assessed by quantifying the proportion of cells

354 with a long flagellum (Fig 6F). We included in this experiment the proteins that were identified

355 only in the LmxFused::BirA* sample with $\geq 4$ spectral counts (LmxM.28.1110, Cilia And

356 Flagella Associated Protein 52 (CFAP52), and LmxM.27.0870 (Component of Motile

357 Flagellum 2 (CMF2)) and those that were highly enriched in LmxFused::BirA* sample

358 compared to the control (spectral index ratio $\geq 10$; LmxM.31.3870 (putative myosin XXI),

359 LmxM.08_29.2030 (putative N-acetylglucosamyl transferase component GPI1), and

360 LmxM.04.0420 (Tetratricopeptide repeat protein)). Loss of myosin XXI, LmxM.04.0420 and

361 LmxM.27.0870 (CMF2) either individually or in combination with $\Delta L m x F u s e d$ did not alter the

362 phenotype of the respective parental cell line (Fig 6F). For GPI1 this could not be assessed,

363 as we were unable to generate a null mutant. By contrast, KO of LmxCFAP52 alone resulted

364 in a $37 \%$ reduction in cells exhibiting long external flagella. Interestingly, the $\Delta L m x F u s e d$ /

$365 \triangle L m x C F A P 52$ dual $\mathrm{KO}$ resulted in cells that lacked long external flagella entirely (Fig 6F),

366 exacerbating the phenotype of the individual gene deletions. Chlamydomonas FAP52 was

367 recently shown to function in the stabilisation of axonemal microtubules (Owa et al., 2019).

368 One possible interpretation of the KO phenotypes observed here in L. mexicana is that 
369 flagellar stability is compromised to different degrees by the loss of CFAP52 and the loss of

370 LmxFused, and when both are lost together, cells can no longer build any flagella.

371

372

373

374

375

376

377

378

379

380

381

382

383

384

385

386

387

388

389

390

391

392

393

394

395

396

397

398

399

400

401

402

403

404

\section{Mammalian ULK4 localises to motile cilia of mouse ependymal cells}

Previous studies have provided several strong lines of evidence linking mammalian Fused/STK36 and ULK4 to motile cilia function (Liu et al., 2016; Wilson et al., 2009) and biochemical evidence indicated proximity of mammalian Fused/STK36 and ULK4 (Preuss et al., 2020). Mammalian Fused/STK36 was shown to localise along the entire length of the motile cilia (Nozawa et al., 2013) but the localization of mammalian ULK4 to motile cilia has not been assessed. We therefore transiently expressed mouse ULK4 (MmULK4) tagged with Flag-HA-mNeonGreen in the primary cell culture of mouse ependymal cells competent of forming motile cilia; aberrant axonemal structure was previously observed in this cell type of Ulk4 hypomorph mice (Liu et al., 2016). Staining fixed cells with an antibody against acetylated tubulin as an axonemal marker, revealed that the tagged MmULK4 localised to multiple foci in the apical region of the cell, in which the cilia originated, and at distal tips of short axonemes (Fig. 7A). An accumulation, albeit with lower signal intensity, was noticeable also at the tips of a subset of longer axonemes. Finally, a weak but discernible signal was observed along each axoneme, and a diffused signal in the cytosol (Fig. 7B).

We next wanted to assess whether the dot-like signals in the apical region could be associated with centrioles. As the combination of the anti-acetylated tubulin antibody and formaldehyde fixation was well suited for the visualisation of axonemes in immunofluorescence, but not for centrioles, we used expansion microscopy, which was previously shown to facilitate access of antibodies to their respective epitopes (Tillberg et al., 2016). Following the ultrastructure expansion microscopy protocol from (Gambarotto et al., 2019) we achieved about 4.7-fold expansion. Indeed, we observed that centrioles in the apical region, which did not extend axonemes, had the Flag-HA-mNeonGreen-MmULK4 associated with them, as determined by anti-HA staining (Fig. 7C). The signal was always juxtaposed distal to one end of the centriole as defined by the anti-acetylated tubulin signal (Fig. 7C). Moreover, expansion microscopy confirmed that Flag-HA-mNeonGreen-MmULK4 was present at the very tips of a subset of long axonemes (Fig. 7D).

Taken together, our data support a function for ULK4 and Fused/STK36 in motile cilia from evolutionarily distant cells, and suggest a model in which ULK4 and Fused/STK36 interact with each other to enable stable assembly of motile flagella and cilia across eukaryotic cells.

\section{Discussion}

Here we characterised the phenotypic consequences of Fused/STK36 and ULK4 knockout on motile flagellum assembly in the protozoan Leishmania mexicana. We found that $\Delta L m x U L K 4$ and $\Delta L m x F u s e d$ cells lines display equivalent flagellar assembly defects, and as 
405

406

407

408

409

410

411

412

413

414

415

416

417

418

419

420

421

422

423

424

425

426

427

428

429

430

431

432

433

434

435

436

437

438

439

440

441

such effectively phenocopy each another (Fig 2 and 3) and suggest they function within the same pathway. Intriguingly, the severity of flagellar structure defects varied between cells within knockout populations (Fig 2, 3 and 4), yet the proportion of distinct flagellar morphotypes remained consistent across multiple independent deletions of both genes and in different parental cell lines (Fig 2, 3 and S6 Fig). These data are consistent with the lossof-function phenotypes described by Baker et al., (Baker et al., 2021) in a knockout screen of Leishmania kinases, and suggest the proteins are involved in regulating ciliogenesis or ciliary stability, but are not essential. In contrast, RNAi mediated knockdown of Fused and ULK4 in the related protist $T$. brucei (Jones et al., 2014; Varga et al., 2017) did not affect flagellar assembly. A small proportion of cells in which FCP6/TbFused was knocked down showed evidence of aberrant cytokinesis, which may be linked to perturbation of motility (Varga et al., 2017). The differences in the severity of phenotype between L. mexicana and T. brucei may be a result of the distinct reverse genetics methods employed (i.e. gene knockout versus knockdown). Alternatively, it could suggest that $T$. brucei possesses an unknown compensatory failsafe mechanism that results in increased flagellar assembly robustness when faced with Fused or ULK4 functional perturbation. A third possibility is that there is some divergence between Fused and ULK4 functions in T. brucei and L. mexicana that could be linked to the differences in cellular architecture. T. brucei flagella have extensive structural connections to the cell body and the newly assembling flagellum is linked to the existing flagellum through the FC structure where Fused or ULK4 were localised. The L. mexicana flagellum is only connected in its proximal part to the to the cell body, via a FAZ structure (Wheeler et al., 2016) and lacks a recognisable FC structure.

In addition to the phenocopy of $\triangle L m x F u s e d$ and $\triangle L m x U L K 4$ described above, we show that LmxFused and LmxULK4 are in close proximity to one another (LmxFused::BirA* mediated BiolD; Supplemental data file 1; Fig 6A), they co-immunoprecipitate (via LmxFused::eYFP pull down; supplemental data file 2; Fig $6 \mathrm{C}$ ), and the stable expression of LmxULK4::mNG is dependent upon the presence of LmxFused (Fig. 6E). Together these data suggest that LmxULK4 and LmxFused function as part of the same pathway to mediate normal motile flagellum assembly or maintenance in Leishmania. Although Fused/STK36 and ULK4 have not yet been shown to directly interact in mammals, both Fused/STK36 and ULK4 mutants are associated with a range of motile cilia assembly defects in distinct mouse tissues (Liu et al., 2016; Nozawa et al., 2013; Vogel et al., 2012; Wilson et al., 2009). Mammalian Fused/STK36 was previously shown to localise to the motile cilium (Nozawa et al., 2013) and we now demonstrated localization of MmULK4 to motile cilia of ependymal cells (Figure 7). Its pattern is indeed reminiscent of the pattern of LmxULK4; MmULK4 localises along the cilium with enrichment at the distal tip of the axoneme. Moreover, the accumulation at tips of nascent cilia resembles the situation in T. brucei, where the ortholog FCP5/TbULK4 localises 
442 to the flagella connector present at the tip of growing flagella (Varga et al., 2017). These

443 finding point to a remarkable conservation of localization of the protein in evolution and make

444 Leishmania a tractable model to dissect the mechanisms by which Fused/STK36 and ULK4

445 contribute to stable flagellum assembly. Interestingly, mammalian ULK4 (tagged with BirA*)

446 has recently been shown to be in close proximity to Fused/STK36 in modified HEK 293T

447 cells (Preuss et al., 2020), supporting the conservation of an evolutionarily ancient

448 Fused/STK36 and ULK4 interaction that likely contributed to the assembly of motile

449 flagella/cilia in the last common ancestor of Leishmania and mammals.

450 To date, relatively little is known about the mechanism through which either protein 451 contribute to flagellar assembly. However, the mouse Fused/STK36 orthologue has been 452 shown to co-immunoprecipitate with the flagella/cilia associated proteins PF20, KIF27 and 453 PCDP1 when expressed in a HEK 293T cell line (Nozawa et al., 2013; Wilson et al., 2009).

454 Of these three established Fused interactors, only the central pair associated protein PF20 455 has an obvious ortholog in L. mexicana. Although LmxPF20 was not shown here to be in 456 close proximity to LmxFused::BirA*, or co-immunoprecipitate with LmxFused::eYFP, we did 457 find that $\triangle L m x U L K 4$ and $\Delta L m x F u s e d$ cells exhibited reduced levels of LmxPF20 in their long 458 external flagella, where present (Fig 3). When considered alongside the other central pair 459 defects noted in $\triangle L m x U L K 4$ and $\triangle L m x F u s e d$ (Fig 3B and 4), these data support the idea that 460 both ULK4 and Fused/STK36 contribute to normal central pair microtubule assembly or 461 maintenance as proposed for mammalian cilia (Liu et al., 2016; Nozawa et al., 2013; Wilson et al., 2009). In some of those mammalian cilia the loss of ULK4 and Fused affected the integrity of the axoneme more widely, however, and in Leishmania, the disruption of the outer doublet microtubule arrangements and reduction of flagellar length were the dominant features of the mutant cells.

466 In addition to LmxULK4, several flagellar proteins were identified within our LmxFused::BirA* BiolD dataset (see supplemental data file 1) as candidate LmxFused interactors. It is interesting to note that CFAP52 and CMF2 were the third and fourth most highly represented proteins identified specifically within the LmxFused::BirA* sample, after only LmxULK4 and the bait proteins itself (Fig 6). Homologs of both CFAP52 (FAP52) and CMF2 (RIB72) in Chlamydomonas are microtubule inner proteins (MIPs) that are bound to inner lumen wall of 472 B and A tubules of the axonemal outer microtubule doublets, respectively (Ma et al., 2019). 473 Both proteins contribute to enhanced microtubule stability against mechanical stress/damage 474 (Owa et al., 2019; Stoddard et al., 2018), and human patients lacking CFAP52 (also known 475 as WDR16) display laterality abnormalities associated with motile cilia dysfunction (e.g. situs 476 inversus totalis (Ta-Shma et al., 2015)). The identification of these proteins in our BiolD 477 dataset suggests that LmxFused::BirA* may directly or indirectly interact with one or multiple 478 MIPs to promote microtubule stabilisation. In support of this hypothesis, we have established 
479 a genetic interaction between LmxCFAP52 and LmxFused, where $\triangle L m x F u s e d$ / $480 \Delta L m x C F A P 52$ cells display an exacerbated flagellar assembly defect relative to $\Delta L m x F u s e d$ 481 alone (they fail to build any long external flagella; see Fig 6F). FAP52 has been described as 482 an interaction hub for a larger MIP network that includes inner junction proteins and also 483 crosses the connection between protofilaments $\mathrm{A} 12$ and $\mathrm{A} 13$ to interact with the $\mathrm{N}$-terminus 484 of RIB72 in the A tubule (Khalifa et al., 2020; Ma et al., 2019). Individual components of this 485 MIP network can act synergistically to promote microtubule stability (e.g. FAP52, FAP45 and 486 FAP20 (Owa et al., 2019)), perhaps warranting the examination of further potential genetic 487 interactions between LmxFused and other MIPs. Though not yet proven, LmxFused could 488 interact with MIPs either prior to their incorporation into the microtubule, via diffusion along 489 the lumen accessed at the microtubule extremities (though the rate of intra-luminal diffusion 490 may be limiting; (Janke and Montagnac, 2017)), or through gaining luminal access at random 491 breakage points to promote microtubule repair. Indeed, it is possible that the diversity of 492 flagellar morphotypes described here are in part a result of the stochastic nature of 493 microtubule destabilisation events that may occur at a higher frequency and/or remain 494 unrepaired in the absence of LmxFused and LmxULK4 associated signalling pathways. It is 495 worth noting that CFAP52 has also been localised to the mouse sperm manchette (Tapia 496 Contreras and Hoyer-Fender, 2020), another microtubular structure that was adversely 497 affected upon the loss of Fused (Nozawa et al., 2014).

498 It should be noted that, apart from LmxULK4, none of the flagellar proteins identified via 499 BiolD also co-immunoprecipitated with LmxFused::eYFP. This discrepancy is likely due to 500 the fact that our IP protocol was biased towards the identification of soluble proteins, as we 501 primarily attempted to confirm the interaction between LmxFused and LmxULK4, both of 502 which appear to be detergent extractable (see S1 Fig). Future efforts to confirm any 503 candidate LmxFused protein kinase substrates identified here could be aided by the 504 generation of a suitable engineered ATP-analogue specific LmxFused kinase (Romano et al., 505 2017).

506 Though not directly tested here, ULK4 is predicted to be a pseudokinase (Fig 1B), similar to 507 the metazoan ULK4 proteins. Interestingly, ULK4 in plants and other protists still possess the 508 canonical catalytically relevant residues and it appears that catalytic activity may have been 509 lost multiple times in evolution (Preuss et al., 2020). LmxFused is predicted to be an active 510 kinase. In Drosophila hedgehog signalling Fused displays both catalytic and non-catalytic 511 functions (see Maloverja \& Piirsoo, (2012) for a review). By contrast, attempts to 512 demonstrate mammalian Fused kinase activity in vitro were unsuccessful (Murone et al., 513 2000; Wilson et al., 2009)), leading to speculation that the mammalian Fused orthologue may 514 not function as an active kinase (Maloverja \& Piirsoo, 2012). However, given the 515 conservation of canonical catalytically relevant amino acid residues within the active site of 
516 these proteins (Fig 1B), and that our results suggest that Fused and ULK4 may function

517 together as a part of the same pathway, it may be interesting to test for mammalian Fused

518 kinase activity in the presence of ULK4.

519 How might ULK4 affect the activity of Fused? Deletions of LmxULK4 KO or LmxFused KO 520 result in the same phenotype with respect to flagellar morphotype (Fig. 2, 3 and 4) In 521 Leishmania the dual $K O$ cell line ( $\Delta L m x U L K 4 / \Delta L m x F u s e d)$ appear phenotypically 522 indistinguishable from either $\Delta L m x U L K 4$ or $\Delta L m x$ Fused (Fig. 2-4). This argues against an 523 inhibitory function of ULK4. Instead ULK4 may stimulate Fused kinase activity in some way; 524 either through the allosteric activation of Fused kinase activity, or functioning as a scaffold to 525 facilitate Fused-substrate interactions which may not otherwise occur at a sufficient rate via 526 diffusion alone (see Figure 8). In Drosophila (which lacks an ULK4 orthologue) Fused 527 homodimerization proceeds trans auto-phosphorylation of the activation loop to promote 528 kinase activity and normal hedgehog signalling (Shi et al., 2011; Zhang et al., 2011). It 529 therefore seems likely that Leishmania orthologue may similarly require both dimerization 530 and activation loop phosphorylation (common protein kinase activation steps) to facilitate 531 LmxFused kinase activity. Indeed, the Fused orthologue in mice appears to oligomerise 532 (Fused proteins tagged with alternate epitopes exhibit co-immunoprecipitation (Nozawa et 533 al., 2013)) supporting the potential for Fused dimerisation (if not multimerization) in mammals 534 as well. Conversely, given the precedent for established pseudokinase-kinase interactions 535 and heterodimers, where the pseudokinase interacting partner regulates the activity of the 536 bona fide catalytic kinase (see (Shaw et al., 2014)) for a review), it is also possible that 537 LmxFused forms a heterodimer with LmxULK4. Shaw et al. (Shaw et al., 2014) hypothesise 538 that pseudokinase-kinase heterodimerization may represent a common phenomenon that 539 facilitates cis autophosphorylation and precedes kinase activation.

540 While this study focused on the role of Fused/STK36 and ULK4 with respect to motile 541 flagellum construction and maintenance, this interaction likely has functional relevance 542 beyond motile cilia and flagella structure and function. In support of this idea, and as 543 highlighted above, ULK4 was shown to be in close proximity to both Fused and several other 544 microtubule associated proteins in HEK 293T cells (Preuss et al., 2020); a cell line which 545 lacks motile cilia. Both proteins also localise to the same layer of the $T$. brucei flagella 546 connector (Varga et al., 2017), though their function within this insoluble mobile 547 transmembrane structure is not yet known. Additionally, although not yet proven 548 experimentally, it has recently been hypothesised that the orthologous proteins of Fused 549 (TIO) and ULK4 (RUK) may cooperate to regulate microtubule depolymerisation in the 550 lagging edge of the cytokinesis/cell plate associated plant phragmoplast (Smertenko et al., 551 2018). The non-ciliated species Dictyostelium has a Fused ortholog called tsunami, which 552 localises to microtubules and tsunami mutants have a chemotaxis and polarisation defect 
553 (Tang et al., 2008). This suggest that interaction with microtubules is an ancestral function of

554 Fused kinases and the interaction between Fused/STK36 and ULK4 appears to represent an

555 evolutionary ancient partnership that likely contributes to the regulation of microtubule

556 stability or microtubule associated signalling pathways in diverse cellular contexts in many

557 eukaryotic organisms.

\section{Materials and Methods}

559 Cell culture and genetic modifications

560 L. mexicana promastigotes (WHO strain MNYC/BZ/62/M379) were cultured at $28^{\circ} \mathrm{C}$ in $\mathrm{M} 199$

561 medium (Life Technologies) supplemented with $2.2 \mathrm{~g} / \mathrm{L} \mathrm{NaHCO} 3,0.005 \%$ haemin, $40 \mathrm{mM}$

562 HEPES and 10\% FCS, pH 7.4 (complete M199). Gene tagging and KO cell lines were

563 generated using CRISPR-Cas9 in the L. mex Cas9 T7 cell line (stabilate Cas9 T7 M), as

564 described by Beneke et al. (Beneke et al., 2017) using primer sequences retrieved from

565 www.LeishGEdit.net (see Beneke \& Gluenz (2019)), unless specified otherwise. Gene KO

566 was confirmed via diagnostic PCR (see primers included in supplemental data file 3 ) used to

567 assess presence or absence of the targeted open reading frame (Beneke \& Gluenz, 2019).

568 Mouse ependymal cell culture was established as described previously (Delgehyr et al.

569 2015). Briefly, the brain of a P1 mouse (C57BI/6J) was dissected. Isolated lateral ventricles

570 were mechanically and enzymatically homogenised, and the culture was expanded. Cells

571 were seeded into a poly-L-lysine (Sigma, P1524) coated 8-well microscopy chamber (Ibidi,

572 80807) for immunofluorescence assay or onto a poly-L-lysine coated $\varnothing 12 \mathrm{~mm}$ microscopy

573 coverslip (Marienfeld, 117520) placed in 24-well plate for expansion microscopy. A plasmid

574 coding for expression of Flag-HA-mNeonGreen-tagged MmULK4 (cDNA: Origene,

575 MR217918; mNeonGreen (mNG) was provided by Allele Biotechnology and Pharmaceuticals

576 (Shaner et al. 2013)) under constitutively active CMV promoter was transfected to the

577 seeded cells using Lipofectamine 3000 (Invitrogen, L3000001) following manufacturer

578 instructions. On the following day, the cells were washed twice with DMEM (Sigma-Aldrich,

579 D6429) supplemented with $100 \mathrm{U} / \mathrm{ml}$ of penicillin (BB Pharma) and $100 \mu \mathrm{g} / \mathrm{ml}$ of streptomycin

580 (Sigma-Aldrich, S9137). The cells were incubated in the serum-free medium for 8 days to

581 induce differentiation and ciliogenesis.

\section{Whole genome sequencing}

583 For the original $\triangle L m x F u s e d$ and $\triangle L m x U L K 4$ cell lines, complete gene $K O$ was also 584 confirmed via whole genome sequencing, as described in (Beneke et al., 2019). Briefly, DNA 585 from mutants was prepared using Illumina TruSeq Nano DNA library kit and resulting 586 libraries were quantified using NEBNext library Quant kit. Library size was determined using 587 Agilent High Sensitivity DNA kit on a 2100 Bioanalyzer instrument. The quantified library was 
588 multiplexed with project unrelated libraries, spiked with 1\% PhiX DNA and sequenced on a

589 NextSeq 550 (1.8 pM loading concentration). Sequencing was performed in paired-end 590 sequencing mode (2x75 cycles, 6 and 8 cycles index read) using a NextSeq 500/550 High

591 Output Kit v2.5 (150 cycles) following the manufactures instructions. Genome samples were 592 de-multiplexed using bcl2fastq (Illumina), assembled using Burrow-Wheeler Aligner (Li and 593 Durbin, 2009), sorted and indexed using Samtools (Li et al., 2009), and viewed on IGV 594 viewer (Robinson et al., 2011).

595 Episomal addback

596 The open reading frame of $L m x F u s e d$ was amplified from genomic DNA using primers

597 F: 5'- TTAGCAACTAGTATGCTTGTGACCATGGAGGACTACC-3' and

598 R: 5'- TTAGCAGAATTCTCAGAGGTCCTCCTCGCTAATGAGCTTTTGCTCCAGGTCTTC 599 TTCGCTGATCAGCTTCTGTTCACCAAGTCGATCGACGAGGTTGC-3'.

600 The resultant PCR products were cloned into pTadd (Beneke et al., 2017), using Spe I and

601 Eco RI restriction sites. The $L m x U L K 4$ addback plasmid was similarly generated using 602 primers

603 F: 5'-TTAGCAACTAGTATGAACAACTATGTGCTTAATGACGAGATC-3' and

$604 \mathrm{R}:$

605 TTTTCAATTGTCAGAGGTCCTCCTCGCTAATGAGCTTTTGCTCCAGGTCTTCTTCGC

606 TGATCAGCTTCTGTTCGGAGAGTTTCTTCAGAATCTTGTTCGC-3', and Spe I and Mfe I

607 restriction sites. $1 \mu \mathrm{g}$ of circular plasmid was transfected as described previously (Beneke et

608 al., 2017) to allow episomal expression of LmxFused::2xMYC and LmxULK4::2xMYC. Drug-

609 resistant cells were selected using $25 \mu \mathrm{g} / \mathrm{ml}$ phleomycin.

\section{Live cell microscopy of Leishmania}

611 Cells were harvested from log phase culture by centrifugation ( $800 \mathrm{~g}, 5$ minutes), washed

612 once in $1 \mathrm{ml}$ PBS (containing, if required, $10 \square \mu \mathrm{g} / \mathrm{ml}$ Hoechst 33342), resuspended in $10 \mu \mathrm{l}$

613 PBS and imaged live, whilst adhered to a poly-lysine coated glass slide. Cells were imaged

614 on either a Zeiss Axioimager.Z2 microscope with a $63 \times$ numerical aperture (NA) 1.40 oil

615 immersion objective and a Hamamatsu ORCA-Flash4.0 camera, or a $63 \times$ NA 1.4 objective

616 lens on a DM5500 B microscope (Leica Microsystems) with a Neo sCMOS camera (Andor

617 Technology) at ambient temperature $\left(\sim 25-28^{\circ} \mathrm{C}\right)$. Micrographs were processed using Fiji

618 (Schindelin et al., 2012).

619 Quantification of IFT train migration and parasite motility analysis

620 IFT fluorescence video micrographs were captured using methods based on those employed 621 by Wheeler at al. (2015), with 600 frames captured per video at $50 \mathrm{~ms}$ fluorescence 622 exposure per frame ( 95 ms delay between frames). IFT particle velocity, intensity and 623 number were quantified using the publicly available software KymographClear (ImageJ 
624 macro toolset) and KymographDirect (see (Mangeol et al., 2016)), including correction for

625 both background signal and signal bleaching. Short particle tracks were removed from the 626 analyses where necessary to avoid pseudo-replication (multiple measurements of an 627 individual particle). This analysis was restricted to cells that possessed a flagellum that 628 extended significantly beyond the flagellar pocket. Promastigote motility assays using 629 darkfield video microscopy were performed using methods described by (Wheeler, 2017), 630 including all three modifications outlined by Beneke et al. (Beneke et al., 2019).

\section{Preparation of cells for immunofluorescence}

632 L. mexicana were harvested from log phase culture by centrifugation $(800 \mathrm{~g}, 5$ minutes), 633 washed twice in $1 \mathrm{ml}$ PBS and resuspended in PBS at $\sim 3 \times 10^{7}$ cells $/ \mathrm{ml}$. Cells were allowed to 634 settle onto a glass slide for 30 minutes in a humid chamber and fixed in cold methanol (20 635 minutes at $-20^{\circ} \mathrm{C}$ ). Samples were rehydrated and washed twice in PBS before antibody 636 labelling. Immunofluorescence assays of mouse cells was performed directly in the 8-well 637 microscopy chamber at room temperature. Cells were washed twice with PBS and fixed with $6384 \%$ formaldehyde (Sigma, F8775) in PBS for 10 minutes. The cells were subsequently 639 washed with PBS and permeabilised with 0.5\% Triton X-100 (Roth, R30512) in PBS for 5 640 minutes, followed by another PBS wash. The permeabilised cells were blocked for 15 641 minutes in a blocking buffer (2\% bovine serum albumin (Roth, 8076.2$), 0.1 \%$ Triton X-100 in 642 PBS), followed by PBS wash.

\section{Antibody labelling and immunofluorescence imaging}

644 The primary antibody C3B9 (mouse monoclonal lgG2b, (Woods et al., 1989) was used for 645 staining acetylated tubulin. The fixed Leishmania were incubated with C3B9 diluted 1:10 in 646 PBS for 1 hour in a humid chamber. The slides were then washed three times (5 minutes per 647 wash) in PBS. Samples were incubated in TRITC-conjugated goat anti-mouse secondary 648 antibody (115-025-146, Jackson ImmunoResearch Laboratories); 1:200 dilution in PBS plus $6495 \%$ goat serum) for 1 hour, and again washed three times (5 minutes per wash) in PBS. 650 Slides were mounted in $90 \%$ glycerol supplemented with $25 \mathrm{mg} / \mathrm{ml} \mathrm{1,4-}$ 651 diazabicyclo[2.2.2]octane (DABCO) and $500 \mathrm{ng} / \mu \mathrm{l}$ diamidino-2-phenylindole (DAPI), $\mathrm{pH}$ 8.6, 652 and imaged via fluorescence light microscopy as described above for live cell microscopy of 653 Leishmania.

654 The fixed mouse cells were incubated with C3B9 diluted 1:50 in blocking buffer for 60 655 minutes. Next, the cells were thoroughly washed with PBS and stained for 30 minutes with 656 Alexa Fluor 647-conjugated goat anti-mouse secondary antibody (A21235, Invitrogen) diluted $657 \quad 1: 1000$ in blocking buffer. Alternatively, the cells were stained with the primary antibody 658 recognizing the ciliary protein Arl13b (17711-1, Proteintech), which was diluted 1:1000 in 659 blocking buffer, followed by staining with Cy5-conjugated goat anti-rabbit (A10523, 
660 Invitrogen) secondary antibody diluted 1:1000 in blocking buffer. The labelled cells were

661 subsequently washed with PBS and stained with $1 \square \mu \mathrm{g} / \mathrm{ml}$ DAPI in PBS for 5 minutes.

662 Finally, the cells were washed with PBS, covered with $120 \mu \mathrm{l}$ of $90 \%$ glycerol supplemented 663 with $\mathrm{DABCO}$, and stored at $4^{\circ} \mathrm{C}$ until imaging.

664 Mouse samples were imaged using a Leica TCS SP8 confocal microscope using an HC PL

665 apochromatic $\times 63 / 1.40$ oil objective. A fluorescence signal was detected using a combination

666 of photomultiplier and hybrid detectors. Z-stacks were deconvolved with Huygens

667 Professional v. 21.04 using deconvolution express mode (Scientific Volume Imaging, The

668 Netherlands, http://svi.nl). Final 3D visualisation was rendered in Imaris viewer 9.7.2

669 (Bitplane). Selected confocal planes were processed using Fiji (Schindelin et al., 2012).

\section{Expansion microscopy}

671 The specimen preparation was based on the U-ExM protocol (Gambarotto et al., 2019) with

672 a few modifications. Briefly, cells were fixed overnight in $4 \%$ formaldehyde and $4 \%$

673 acrylamide (Sigma, A8887) in PBS at room temperature. For gelation, the cells were

674 incubated for 30 minutes at $37^{\circ} \mathrm{C}$ in a humidified incubator in $50 \mu$ of monomer solution 675 containing 19\% sodium acrylate (Sigma, 408220), 10\% acrylamide, $0.1 \% \quad \mathrm{~N}, \quad \mathrm{~N}$ '676 methylenebisacrylamide (Sigma, M7256), 0.5\% N, N, N', N'-tetramethylethylenediamine 677 (Sigma, T9281) and 0.5\% ammonium persulfate (Thermo Scientific, 17874). After 678 denaturation $\left(60\right.$ minutes at $95^{\circ} \mathrm{C}$ in denaturation buffer composed of $50 \mathrm{mM}$ Tris, $200 \mathrm{mM}$ $679 \mathrm{NaCl}$, and $200 \mathrm{mM}$ sodium dodecyl sulfate in $\mathrm{dd}_{2} \mathrm{O} ; \mathrm{pH}$ 9.0) and expansion of the gel by 680 incubation in ultrapure water, $1 \times 1 \mathrm{~cm}$ piece of the gel was cut and incubated overnight with 681 primary antibodies (C3B9 (Woods et al., 1989) diluted 1:10, and an anti-HA tag antibody 682 (Cell Signaling Technology, 3724S) diluted 1:500 in 2\% bovine serum albumin in PBS). After $6833 \times 20$ minute washes with ultrapure water, the gel was stained overnight with secondary 684 antibodies (Alexa Fluor 488 anti-mouse (Invitrogen, A11001) diluted 1:500, and Alexa Fluor 685555 anti-rabbit (Invitrogen, A21428) diluted 1:500 in 2\% bovine serum albumin in PBS). 686 Finally, the piece of gel was washed $3 \times 20$ minutes and stored in ultrapure water until 687 imaging. The expanded gel was placed on a poly-L-lysine coated glass-bottom dish (Cellvis, 688 D35-20-1.5-N) and imaged as described above.

\section{Transmission electron microscopy}

690 Cells were prepared with a chemical fixation protocol similar to that outlined by Hoog et al. 691 (Hoog et al., 2010). Briefly, cells were fixed with 2.5\% glutaraldehyde and 4\% 692 paraformaldehyde in complete M199 culture medium for 2 hours at room temperature. Fixed 693 cells were washed six times for 10 minutes in $0.1 \mathrm{M}$ piperazine-N,N'-bis(2-ethanesulfonic 694 acid) buffer (PIPES, pH 7.2), with the forth wash supplemented with $50 \mathrm{mM}$ glycine. Cells 695 were embedded in 3\% low-melting-point agar and incubated in 1\% osmium tetroxide and 
$6961.5 \%$ potassium ferrocyanide in $0.1 \mathrm{M}$ PIPES buffer rotating in darkness at $4^{\circ} \mathrm{C}$ for 1 hour.

697 Samples were then washed five times with $\mathrm{ddH}_{2} \mathrm{O}$ (5 minutes per wash), and stained with

$6980.5 \%$ uranyl acetate at $4^{\circ} \mathrm{C}$ overnight in darkness. Samples were dehydrated, embedded in 699 epoxy resin, sectioned and on section stained as described previously (Hoog et al., 2010).

700 Electron micrographs were captured on a Tecnai 12 TEM (FEI) with an Ultrascan 1000 CCD

701 camera (Gatan), and processed using Fiji (Schindelin et al., 2012).

\section{Biotin ligase mediated proximity labelling}

703 A cell line expressing LmxFused::BirA* was generated by tagging both $L m x F u s e d$ alleles 704 with pPLOT-BirA*::3xmyc (Beneke et al., 2017), and selected on $20 \mu \mathrm{g} / \mathrm{ml}$ puromycin. Clonal 705 cell lines were selected by limiting dilution and the modification of both alleles was confirmed 706 via endpoint PCR (using primers that spanned the inserted Bir $A^{*}$ tag encoding region; F: 5'707 CGGGGCACTGAGCAACTTTGT-3', R: 5'-CACCAGTGGGCAGTGTGAGC-3'). Biotinylated 708 proteins were captured using the XL-BiolD protocol, which includes a cross-linking step prior 709 to affinity capture (Geoghegan et al., 2021): Log phase promastigote cultures (seeded at $7102 \times 10^{6} \mathrm{cell} / \mathrm{ml}$ ) were allowed to grow in complete M199 supplemented with $150 \mu \mathrm{M}$ biotin (B7114639 , Sigma-Aldrich) for $18-24$ hours. $4 \times 10^{8}$ parasites were harvested via centrifugation 712 (800g for 5 minutes), washed twice with PBS, and cross-linked with $1 \mathrm{mM}$ 713 dithiobis(succinimidyl propionate) (DSP) in $10 \mathrm{ml}$ PBS plus $5 \%$ dimethyl sulfoxide (DMSO) 714 for 10 minutes at $28^{\circ} \mathrm{C}$. $1 \mathrm{M}$ Tris $\mathrm{pH} 7.5$ was added for 5 minutes at room temperature to 715 quench, and parasites were pelleted via centrifugation (1200g, 3 minutes). All subsequent 716 steps were performed on ice or at $4^{\circ} \mathrm{C}$. The supernatant was discarded and parasites lysed 717 with sterile RIPA buffer plus protease and phosphatase inhibitors (1\% NP-40, 0.5\% sodium 718 deoxycholate, $0.1 \%$ SDS, $50 \mathrm{mM}$ Tris $\mathrm{pH}$ 7.5, $125 \mathrm{mM} \mathrm{NaCl}, 0.1 \mathrm{mM}$ EDTA, $0.1 \mathrm{mM}$ 719 Phenylmethanesulfonyl fluoride (PMSF), $1 \mu \mathrm{g} / \mathrm{ml}$ Pepstatin A, $1 \mu \mathrm{M}$ trans-Epoxysuccinyl-L720 leucylamido(4-guanidino)butane (E-64), 0.4 mM 1-10 phenanthroline, 1 tablet/ml cOmplete ${ }^{\mathrm{TM}}$ 721 inhibitor cocktail (Roche), 1x PhosSTOP ${ }^{\mathrm{TM}}$ (Roche) and 1x Halt ${ }^{\mathrm{TM}}$ Proteases inhibitors 722 single-use cocktail (Thermo Scientific)), and sonicated (three times for 5 seconds at 723 amplitude 16 on a MSE Soniprep 150). Benzonase (BaseMuncher; 0.5 units/ $/$ l) was added 724 to digest chromatin for 1 hour and the lysate was centrifuged at $10^{\prime} 000 \mathrm{~g}$ for 10 minutes at $7254^{\circ} \mathrm{C}$. The supernatant was added to $1 \mathrm{mg}$ MagResyn® streptavidin beads (MR-STV002, 726 Resyn Biosciences); note that these beads were washed twice with $1 \mathrm{ml}$ of RIPA buffer 727 before use and rotated overnight to enable biotinylated protein binding. The beads were then 728 washed sequentially in $500 \mu \mathrm{l}$ of RIPA buffer (4 washes in total), followed by individual 729 washes in $4 \mathrm{M}$ urea, $6 \mathrm{M}$ urea and $1 \mathrm{M} \mathrm{KCl}$. The beads were resuspended in $50 \mathrm{mM}$ TEAB 730 (triethylammonium bicarbonate). Samples capturing an equivalent fraction of the input 731 material were collected at multiple points during the protocol, and SDS-PAGE and 
732 immunoblots were performed by standard methods using a mouse monoclonal anti-MYC

733 antibody (clone 4A6, 05-724, Merck).

734

735

736

737

738

739

740

741

742

743

744

745

746

747

748

749

750

751

752

753

754

755

756

757

758

759

760

761

762

763

764

765

766
Mass spectrometry for BiolD

Sample preparation and analysis was performed by the Advanced Proteomics Facility (Department of Biochemistry, University of Oxford) as follows: On-bead protein sample digests were performed according to the filter-aided sample preparation procedure described in (Wisniewski et al., 2009). Peptides were analysed by nano-liquid chromatography tandem mass spectrometry (nano-LC/MS/MS) on an Orbitrap $Q$ Exactive mass spectrometer (Thermo Scientific) using Higher-energy Collisional Dissociation (HCD) fragmentation. In brief, peptides were loaded on a C18 PepMap100 pre-column $(300 \mu \mathrm{m}$ i.d. x $5 \mathrm{~mm}, 100 \AA$ (Thermo Fisher Scientific)) at a flow rate of $12 \mu \mathrm{l} / \mathrm{min}$ in $100 \%$ buffer $\mathrm{A}(0.1 \%$ formic acid in $\left.\mathrm{H}_{2} \mathrm{O}\right)$. Peptides were then transferred to an in-house packed analytical column heated at $45^{\circ} \mathrm{C}(50 \mathrm{~cm}, 75 \mu \mathrm{m}$ i.d. packed with ReproSil-Pur $120 \mathrm{C} 18-\mathrm{AQ}, 1.9 \mu \mathrm{m}, 120 \AA$ ) and separated using a 60 minute gradient from 15 to $35 \%$ buffer $B(0.1 \%$ formic acid in acetonitrile) at a flow rate of $200 \mathrm{nl} / \mathrm{min}$. Q Exactive survey scans were acquired at 70,000 resolution to a scan range from 350 to $1500 \mathrm{~m} / \mathrm{z}$, automatic gain control target 3e6, maximum injection time $50 \mathrm{~ms}$. The mass spectrometer was operated in a data-dependent mode to automatically switch between MS and MS/MS. The 10 most intense precursor ions were submitted to HCD fragmentation using an MS/MS resolution set to 17500 , a precursor automatic gain control target set to $5 \mathrm{e} 4$, a precursor isolation width set to $1.5 \mathrm{Da}$, and a maximum injection time set to $120 \mathrm{~ms}$. The data was converted from .raw to .mgf file formats using ProteoWizard and analyzed through the Advanced Proteomics Facility Pipeline (CPFP; see (Trudgian et al., 2010)). A list of proteins was generated using CPFP meta-search Simple Protein Overview tool, using L. mexicana proteome as reference (gene models based on (Fiebig et al., 2015)) and label-free SINQ quantification, using search parameters outlined by (Beneke et al., 2019). Proteins were filtered based on a $5 \%$ false discovery rate (FDR) and the identification of at least 2 unique peptides.

\section{Immunoprecipitation}

Cell lines expressing $L m x F u s e d:: e Y F P$ or $L m x U L K 4:: 3 x M Y C$ were generated by tagging both alleles of the target gene with eYFP or 3XMYC, respectively, using the LeishGEdit method (Beneke et al., 2017). Specific primers were designed for amplification of donor DNA cassettes, as follows. To tag the C-terminus of $L m x U L K 4$ with three MYC epitope tags, donor PCR products amplified from the standard pPLOT plasmids;

F 5'-GAGGCGAACAAGATTCTGAAGAAACTCTCCGGATCCGGATCAGGATCTGG-3' R 5'-AAATCGTACAGGCAACAGCAAACCCGCCCGCCAATTTGAGAGACCTGTGC-3'. 
767 To generate donor PCR products for LmxFused C-terminal tagging with an eYFP tag without

768 a MYC epitope, eYFP was amplified from the pJ1170/pLEnT-YB plasmid (Dean et al., 2015):

769 F 5'-TACGTTGGCAACCTCGTCGATCGACTTGGTGGTTCTGGTAGTGGTTCCGGTTCCGGTT

770 CTGTGAGCAAGGGCGAGGAGCTGTT-3'

771 R 5'-AAATCTGGAAAACGGCAACTATGAAGTCCGTTAGCCCTCCCACACATAACCAGAG-3').

772 Immunoprecipitation with anti-GFP antibodies followed the protocol from Akiyoshi \& Gull

773 (2014), with the following modifications: Instead of using PEME buffer, cells were extracted

774 directly in modified buffer $\mathrm{H}(\mathrm{BH}) / 0.15$ (25 mM HEPES pH 8.0, $2 \mathrm{mM} \mathrm{MgCl}, 0.1 \mathrm{mM}$ EDTA

$775 \mathrm{pH} 8.0,0.5 \mathrm{mM}$ EGTA pH 8.0, $1 \% \mathrm{NP}-40,150 \mathrm{mM} \mathrm{KCl}$, and $15 \%$ glycerol), including

776 protease inhibitors (Leupeptin, Pepstatin, E-64, $20 \mu \mathrm{g} / \mathrm{ml}$ each, and $0.2 \mathrm{mM} \mathrm{PMSF}$ ) and

777 phosphatase inhibitors (1 $\mathrm{mM}$ sodium pyrophosphate, $2 \mathrm{mM}$ Na-beta-glycerophosphate, 0.1

$778 \mathrm{mM} \mathrm{Na}_{3} \mathrm{VO}_{4}, 5 \mathrm{mM} \mathrm{NaF}$, and $100 \mathrm{nM}$ microcystin-LR). Cell extracts were frozen in liquid $\mathrm{N}_{2}$

779 and ground in a mortar and pestle, without sonication. Cell extracts were then centrifuged

$780\left(14000 \mathrm{~g}\right.$ for 30 minutes at $\left.4^{\circ} \mathrm{C}\right)$ and only the supernatant was forwarded to

781 immunoprecipitation. Post-IP bead samples were then washed twice in PBS and eluted in

$7820.2 \mathrm{M}$ Glycine, $\mathrm{pH} 2.5$ (shaking at $21^{\circ} \mathrm{C}$ for 7 minutes) and neutralised with $10 \%$ volume $1 \mathrm{M}$

783 Tris- $\mathrm{HCl}, \mathrm{pH}$ 8.8. Equivalent proportions of each sample were collected at multiple points

784 during the protocol, with SDS-PAGE and immunoblots performed by standard methods using

785 the following mouse monoclonal antibodies: anti-GFP (11814460001, Roche) and anti-MYC

786 (clone 4A6, 05-724, Merck).

787

\section{LC-MS/MS for IP/Immunoprecipitation}

788 Mass spectrometry analysis was performed by the Advanced Proteomics facility (Department

789 of Biochemistry, University of Oxford). Peptides were separated by nano liquid

790 chromatography (Thermo Scientific Ultimate RSLC 3000) coupled in line a Q Exactive mass

791 spectrometer equipped with an Easy-Spray source (Thermo Fischer Scientific). Peptides

792 were trapped onto a C18 PepMac100 precolumn $(300 \mu \mathrm{m}$ i.d.x5mm, 100Å, ThermoFischer

793 Scientific) using Solvent A (0.1\% Formic acid, HPLC grade water). The peptides were further

794 separated onto an Easy-Spray RSLC C18 column (75um i.d., $50 \mathrm{~cm}$ length, Thermo Fischer

795 Scientific) using a 60 minutes linear gradient (15\% to $35 \%$ solvent B $(0.1 \%$ formic acid in

796 acetonitrile)) at a flow rate $200 \mathrm{nl} / \mathrm{min}$. The raw data were acquired on the mass spectrometer

797 in a data-dependent acquisition mode (DDA). Full-scan MS spectra were acquired in the

798 Orbitrap (Scan range 350-1500m/z, resolution 70,000; AGC target, 3e6, maximum injection

799 time, 50ms). The 10 most intense peaks were selected for higher-energy collision

800 dissociation (HCD) fragmentation at $30 \%$ of normalized collision energy. HCD spectra were

801 acquired in the Orbitrap at resolution 17,500, AGC target 5e4, maximum injection time

$802120 \mathrm{~ms}$ with fixed mass at $180 \mathrm{~m} / \mathrm{z}$. Charge exclusion was selected for unassigned and $1+$

803 ions. The dynamic exclusion was set to $20 \mathrm{~s}$. 


\section{Acknowledgements}

805 We thank Sabrina Liberatori and Marjorie Fournier (University of Oxford Proteomics facility) 806 for mass spectrometry support, Raman Dhaliwal and Errin Johnson (Dunn School 807 Bioimaging facility) for electron microscopy support, James Smith (University of Oxford) for 808 help with the generation of Leishmania mutants, Richard Wheeler (University of Oxford) for 809 advice on IFT measurements, Bungo Akiyoshi (University of Oxford) for advice on the IP

810 protocol, Alice Meunier and Nathalie Spassky (ENS Paris) for introducing us to preparation of 811 ependymal cell cultures, Jeremy Mottram and Vincent Geoghegan (University of York) for 812 sharing their XL-BioID protocol prior to publication and Keith Gull (University of Oxford) for 813 antibody C3B9, access to laboratory equipment and helpful comments on the manuscript.

\section{Competing Interests}

815 No competing interests declared

\section{Funding}

817 CMC and EG were funded by the UK Medical Research Council (MRC) and the UK 818 Department for International Development (DFID) under the MRC/DFID Concordat 819 agreement; grant no. MR/R000859/1 (https://mrc.ukri.org/).

820 EG was supported by a Royal Society University Research Fellowship (UF160661; 821 https://royalsociety.org/)

822 HPV was supported by an ERASMUS+ mobility grant

823 TB was supported by MRC PhD studentship (15/16_MSD_836338)

824 VV laboratory was supported by the Czech Science Foundation (GA CR) project no. 20$82523165 \mathrm{~J}$ and by an Installation Grant from the European Molecular Biology Organization.

826 PG is a student of the Faculty of Science, Charles University, Prague, which provided a PhD 827 student fellowship.

828 We acknowledge support for this project for EG through the WCIP core Wellcome Centre 829 Award [104111/Z/14/Z] and through the Wellcome Trust grant [104627/Z/14/Z] to Keith Gull; 830 https://wellcome.org).

831 We acknowledge the Light Microscopy Core Facility, IMG CAS, Prague, Czech Republic, 832 supported by MEYS (LM2018129, CZ.02.1.01/0.0/0.0/18_046/0016045) and RVO: $83368378050-K A V-N P U I$, for their support with the confocal imaging of ependymal cells.

834 The funders had no role in study design, data collection and analysis, decision to publish, or 835 preparation of the manuscript. 
837

838

839

840

841

842

843

844

845

846

847

848

849

850

851

852

853

854

855

856

857

858

859

860

861

862

863

864

865

866

867

868

869

870

871

872 Hoog, J.L., Gluenz, E., Vaughan, S., and Gull, K. (2010). Ultrastructural investigation 873 methods for Trypanosoma brucei. Methods in Cell Biology 96, 175-196. 874 Janke, C., and Montagnac, G. (2017). Causes and Consequences of Microtubule 875 Acetylation. Curr Biol 27, R1287-R1292.

Amos, B., Aurrecoechea, C., Barba, M., Barreto, A., Basenko, E.Y., Bazant, W., Belnap, R., Blevins, A.S., Bohme, U., Brestelli, J., et al. (2021). VEuPathDB: the eukaryotic pathogen, vector and host bioinformatics resource center. Nucleic Acids Research 50, D898-D911.

Baker, N., Catta-Preta, C.M.C., Neish, R., Sadlova, J., Powell, B., Alves-Ferreira, E.V.C., Geoghegan, V., Carnielli, J.B.T., Newling, K., Hughes, C., et al. (2021). Systematic functional analysis of Leishmania protein kinases identifies regulators of differentiation or survival. Nat Commun 12, 1244.

Beneke, T., Demay, F., Hookway, E., Ashman, N., Jeffery, H., Smith, J., Valli, J., Becvar, T., Myskova, J., Lestinova, T., et al. (2019). Genetic dissection of a Leishmania flagellar proteome demonstrates requirement for directional motility in sand fly infections. PLoS Pathogens 15, e1007828.

Beneke, T., Madden, R., Makin, L., Valli, J., Sunter, J., and Gluenz, E. (2017). A CRISPR Cas9 high-throughput genome editing toolkit for kinetoplastids. Royal Society Open Science 4, 170095.

Chen, M.H., Gao, N., Kawakami, T., and Chuang, P.T. (2005). Mice deficient in the fused homolog do not exhibit phenotypes indicative of perturbed hedgehog signaling during embryonic development. Molecular and Cellular Biology 25, 7042-7053.

Dean, S., Sunter, J., Wheeler, R.J., Hodkinson, I., Gluenz, E., and Gull, K. (2015). A toolkit enabling efficient, scalable and reproducible gene tagging in trypanosomatids. Open Biology $5,140197$.

Edelbusch, C., Cindric, S., Dougherty, G.W., Loges, N.T., Olbrich, H., Rivlin, J., Wallmeier, J., Pennekamp, P., Amirav, I., and Omran, H. (2017). Mutation of serine/threonine protein kinase 36 (STK36) causes primary ciliary dyskinesia with a central pair defect. Hum Mutat 38, 964-969.

Fiebig, M., Kelly, S., and Gluenz, E. (2015). Comparative lifecycle transcriptomics revises Leishmania mexicana genome annotation and links a chromosome duplication with parasitism of vertebrates. PLoS Pathogens 11, e1005186.

Gambarotto, D., Zwettler, F.U., Le Guennec, M., Schmidt-Cernohorska, M., Fortun, D., Borgers, S., Heine, J., Schloetel, J.G., Reuss, M., Unser, M., et al. (2019). Imaging cellular ultrastructures using expansion microscopy (U-ExM). Nature Methods 16, 71-74.

Geoghegan, V., Jones, N.G., Dowle, A., and Mottram, J.C. (2021). Protein kinase signalling at the Leishmania kinetochore captured by XL-BioID. bioRxiv, 2021.2007.2008.451598.

Goetz, S.C., and Anderson, K.V. (2010). The primary cilium: a signalling centre during vertebrate development. Nature Reviews Genetics 11, 331-344. 
876 Jones, N.G., Thomas, E.B., Brown, E., Dickens, N.J., Hammarton, T.C., and Mottram, J.C. (2014). Regulators of Trypanosoma brucei cell cycle progression and differentiation identified using a kinome-wide RNAi screen. PLoS Pathogens 10, e1003886.

879 Khalifa, A.A.Z., Ichikawa, M., Dai, D., Kubo, S., Black, C.S., Peri, K., McAlear, T.S., Veyron, 880 S., Yang, S.K., Vargas, J., et al. (2020). The inner junction complex of the cilia is an 881 interaction hub that involves tubulin post-translational modifications. eLife 9.

882 Kumar, S., Stecher, G., and Tamura, K. (2016). MEGA7: Molecular Evolutionary Genetics 883 Analysis Version 7.0 for Bigger Datasets. Molecular Biology and Evolution 33, 1870-1874.

884 Lang, B., Zhang, L., Jiang, G., Hu, L., Lan, W., Zhao, L., Hunter, I., Pruski, M., Song, N.N., 885 Huang, Y., et al. (2016). Control of cortex development by ULK4, a rare risk gene for mental 886 disorders including schizophrenia. Scientific Reports 6, 31126.

887 Lechtreck, K.F. (2015). IFT-Cargo Interactions and Protein Transport in Cilia. Trends 888 Biochem Sci 40, 765-778.

889 Lee, L. (2011). Mechanisms of mammalian ciliary motility: Insights from primary ciliary 890 dyskinesia genetics. Gene 473, 57-66.

$891 \mathrm{Li}, \mathrm{H}$., and Durbin, R. (2009). Fast and accurate short read alignment with Burrows-Wheeler 892 transform. Bioinformatics 25, 1754-1760.

893 Li, H., Handsaker, B., Wysoker, A., Fennell, T., Ruan, J., Homer, N., Marth, G., Abecasis, G., 894 Durbin, R., and Genome Project Data Processing, S. (2009). The Sequence Alignment/Map 895 format and SAMtools. Bioinformatics 25, 2078-2079.

896 Liu, M., Guan, Z., Shen, Q., Lalor, P., Fitzgerald, U., O'Brien, T., Dockery, P., and Shen, S. 897 (2016). Ulk4 Is Essential for Ciliogenesis and CSF Flow. J Neurosci 36, 7589-7600.

898 Ma, M., Stoyanova, M., Rademacher, G., Dutcher, S.K., Brown, A., and Zhang, R. (2019). 899 Structure of the Decorated Ciliary Doublet Microtubule. Cell 179, 909-922 e912.

900 Maloverjan, A., Piirsoo, M., Kasak, L., Peil, L., Osterlund, T., and Kogerman, P. (2010). Dual 901 function of UNC-51-like kinase 3 (Ulk3) in the Sonic hedgehog signaling pathway. The 902 Journal of Biological Chemistry 285, 30079-30090.

903 Mangeol, P., Prevo, B., and Peterman, E.J. (2016). KymographClear and KymographDirect: 904 two tools for the automated quantitative analysis of molecular and cellular dynamics using 905 kymographs. Molecular Biology of the Cell 27, 1948-1957.

906 Merchant, M., Evangelista, M., Luoh, S.M., Frantz, G.D., Chalasani, S., Carano, R.A., van 907 Hoy, M., Ramirez, J., Ogasawara, A.K., McFarland, L.M., et al. (2005). Loss of the 908 serine/threonine kinase fused results in postnatal growth defects and lethality due to 909 progressive hydrocephalus. Molecular and Cellular Biology 25, 7054-7068.

910 Moreira-Leite, F.F., Sherwin, T., Kohl, L., and Gull, K. (2001). A trypanosome structure 911 involved in transmitting cytoplasmic information during cell division. Science 294, 610-612.

912 Murone, M., Luoh, S.M., Stone, D., Li, W., Gurney, A., Armanini, M., Grey, C., Rosenthal, A., 913 and de Sauvage, F.J. (2000). Gli regulation by the opposing activities of fused and 914 suppressor of fused. Nature Cell Biology 2, 310-312.

915 Nakayama, K., and Katoh, Y. (2018). Ciliary protein trafficking mediated by IFT and BBSome 916 complexes with the aid of kinesin-2 and dynein-2 motors. J Biochem 163, 155-164. 
917 Nozawa, Y.I., Yao, E., Gacayan, R., Xu, S.M., and Chuang, P.T. (2014). Mammalian Fused

918 is essential for sperm head shaping and periaxonemal structure formation during

919 spermatogenesis. Dev Biol 388, 170-180.

920 Nozawa, Y.I., Yao, E., Lin, C., Yang, J.H., Wilson, C.W., Gacayan, R., and Chuang, P.T.

921 (2013). Fused (Stk36) is a ciliary protein required for central pair assembly and motile cilia

922 orientation in the mammalian oviduct. Dev Dyn 242, 1307-1319.

923 Owa, M., Uchihashi, T., Yanagisawa, H.A., Yamano, T., Iguchi, H., Fukuzawa, H.,

924 Wakabayashi, K.I., Ando, T., and Kikkawa, M. (2019). Inner lumen proteins stabilize doublet

925 microtubules in cilia and flagella. Nat Commun 10, 1143.

926 Portran, D., Schaedel, L., Xu, Z., Thery, M., and Nachury, M.V. (2017). Tubulin acetylation

927 protects long-lived microtubules against mechanical ageing. Nature Cell Biology 19, 391-398.

928 Preat, T., Therond, P., Lamour-Isnard, C., Limbourg-Bouchon, B., Tricoire, H., Erk, I., Mariol, 929 M.C., and Busson, D. (1990). A putative serine/threonine protein kinase encoded by the 930 segment-polarity fused gene of Drosophila. Nature 347, 87-89.

931 Preuss, F., Chatterjee, D., Mathea, S., Shrestha, S., St-Germain, J., Saha, M., Kannan, N., 932 Raught, B., Rottapel, R., and Knapp, S. (2020). Nucleotide Binding, Evolutionary Insights, 933 and Interaction Partners of the Pseudokinase Unc-51-like Kinase 4. Structure 28, 1184-1196 934 e1186.

935 Ringers, C., Olstad, E.W., and Jurisch-Yaksi, N. (2020). The role of motile cilia in the 936 development and physiology of the nervous system. Philosophical transactions of the Royal Society of London Series B, Biological Sciences 375, 20190156.

938 Robinson, J.T., Thorvaldsdottir, H., Winckler, W., Guttman, M., Lander, E.S., Getz, G., and 939 Mesirov, J.P. (2011). Integrative genomics viewer. Nature Biotechnology 29, 24-26.

940 Romano, V., de Beer, T.A., and Schwede, T. (2017). A computational protocol to evaluate 941 the effects of protein mutants in the kinase gatekeeper position on the binding of ATP 942 substrate analogues. BMC Res Notes 10, 104.

943 Roux, K.J., Kim, D.I., Raida, M., and Burke, B. (2012). A promiscuous biotin ligase fusion 944 protein identifies proximal and interacting proteins in mammalian cells. The Journal of Cell 945 Biology 196, 801-810.

946 Schindelin, J., Arganda-Carreras, I., Frise, E., Kaynig, V., Longair, M., Pietzsch, T., 947 Preibisch, S., Rueden, C., Saalfeld, S., Schmid, B., et al. (2012). Fiji: an open-source 948 platform for biological-image analysis. Nature Methods 9, 676-682.

949 Shaw, A.S., Kornev, A.P., Hu, J., Ahuja, L.G., and Taylor, S.S. (2014). Kinases and 950 pseudokinases: lessons from RAF. Molecular and Cellular Biology 34, 1538-1546.

951 Shi, Q., Li, S., Jia, J., and Jiang, J. (2011). The Hedgehog-induced Smoothened 952 conformational switch assembles a signaling complex that activates Fused by promoting its 953 dimerization and phosphorylation. Development 138, 4219-4231.

954 Smertenko, A., Hewitt, S.L., Jacques, C.N., Kacprzyk, R., Liu, Y., Marcec, M.J., Moyo, L., 955 Ogden, A., Oung, H.M., Schmidt, S., et al. (2018). Phragmoplast microtubule dynamics - a 956 game of zones. Journal of Cell Science 131.

957 Stoddard, D., Zhao, Y., Bayless, B.A., Gui, L., Louka, P., Dave, D., Suryawanshi, S., Tomasi, 958 R.F., Dupuis-Williams, P., Baroud, C.N., et al. (2018). Tetrahymena RIB72A and RIB72B are 
959 microtubule inner proteins in the ciliary doublet microtubules. Molecular Biology of the Cell 29, 2566-2577.

961 Ta-Shma, A., Perles, Z., Yaacov, B., Werner, M., Frumkin, A., Rein, A.J., and Elpeleg, O. 962 (2015). A human laterality disorder associated with a homozygous WDR16 deletion. Eur J 963 Hum Genet 23, 1262-1265.

964 Tang, L., Franca-Koh, J., Xiong, Y., Chen, M.Y., Long, Y., Bickford, R.M., Knecht, D.A., 965 Iglesias, P.A., and Devreotes, P.N. (2008). tsunami, the Dictyostelium homolog of the Fused 966 kinase, is required for polarization and chemotaxis. Genes \& Development 22, 2278-2290.

967 Tapia Contreras, C., and Hoyer-Fender, S. (2020). The WD40-protein CFAP52/WDR16 is a 968 centrosome/basal body protein and localizes to the manchette and the flagellum in male 969 germ cells. Scientific Reports 10, 14240.

970 Tillberg, P.W., Chen, F., Piatkevich, K.D., Zhao, Y., Yu, C.C., English, B.P., Gao, L., Martorell, A., Suk, H.J., Yoshida, F., et al. (2016). Protein-retention expansion microscopy of cells and tissues labeled using standard fluorescent proteins and antibodies. Nature Biotechnology 34, 987-992.

974 Trudgian, D.C., Ridlova, G., Fischer, R., Mackeen, M.M., Ternette, N., Acuto, O., Kessler,

975 B.M., and Thomas, B. (2011). Comparative evaluation of label-free SINQ normalized spectral 976 index quantitation in the central proteomics facilities pipeline. Proteomics 11, 2790-2797.

977 Trudgian, D.C., Thomas, B., McGowan, S.J., Kessler, B.M., Salek, M., and Acuto, O. (2010). 978 CPFP: a central proteomics facilities pipeline. Bioinformatics 26, 1131-1132.

979 UniProt, C. (2021). UniProt: the universal protein knowledgebase in 2021. Nucleic Acids 980 Research 49, D480-D489.

981 Varga, V., Moreira-Leite, F., Portman, N., and Gull, K. (2017). Protein diversity in discrete 982 structures at the distal tip of the trypanosome flagellum. Proceedings of the National 983 Academy of Sciences of the United States of America 114, E6546-E6555.

984 Vogel, P., Read, R.W., Hansen, G.M., Payne, B.J., Small, D., Sands, A.T., and Zambrowicz, 985 B.P. (2012). Congenital hydrocephalus in genetically engineered mice. Vet Pathol 49, 166986181.

987 Wheeler, R.J. (2017). Use of chiral cell shape to ensure highly directional swimming in 988 trypanosomes. PLoS Computational Biology 13, e1005353.

989 Wheeler, R.J., Sunter, J.D., and Gull, K. (2016). Flagellar pocket restructuring through the 990 Leishmania life cycle involves a discrete flagellum attachment zone. Journal of Cell Science 991 129, 854-867.

992 Wilson, C.W., Nguyen, C.T., Chen, M.H., Yang, J.H., Gacayan, R., Huang, J., Chen, J.N., 993 and Chuang, P.T. (2009). Fused has evolved divergent roles in vertebrate Hedgehog 994 signalling and motile ciliogenesis. Nature 459, 98-102.

995 Wisniewski, J.R., Zougman, A., Nagaraj, N., and Mann, M. (2009). Universal sample 996 preparation method for proteome analysis. Nature Methods 6, 359-362.

997 Woods, A., Sherwin, T., Sasse, R., MacRae, T.H., Baines, A.J., and Gull, K. (1989). 998 Definition of individual components within the cytoskeleton of Trypanosoma brucei by a 999 library of monoclonal antibodies. Journal of Cell Science 93 ( Pt 3), 491-500. 
bioRxiv preprint doi: https://doi.org/10.1101/2022.03.06.483169; this version posted March 6, 2022. The copyright holder for this preprint (which was not certified by peer review) is the author/funder, who has granted bioRxiv a license to display the preprint in perpetuity. It is made available under aCC-BY 4.0 International license.

1000 Zhang, Y., Mao, F., Lu, Y., Wu, W., Zhang, L., and Zhao, Y. (2011). Transduction of the 1001 Hedgehog signal through the dimerization of Fused and the nuclear translocation of Cubitus 1002 interruptus. Cell Res 21, 1436-1451.

1003 Zhou, Q., An, T., Pham, K.T.M., Hu, H., and Li, Z. (2018). The CIF1 protein is a master 1004 orchestrator of trypanosome cytokinesis that recruits several cytokinesis regulators to the 1005 cytokinesis initiation site. The Journal of Biological Chemistry 293, 16177-16192.

1006 


\section{Figure Legends}

1008

1009

1010

1011

1012

1013

1014

1015

1016

1017

1018

1019

1020

1021

1022

1023

1024

1025

1026

1027

1028

1029

1030

1031

1032

1033

1034

1035

1036

1037

1038

1039

1040

1041

1042

1043

1044

1045

1046

1047

1048

1049

1050

1051

Fig 1. UNC-51 like kinase orthologs in L. mexicana and their subcellular localisation. (A) Position of L. mexicana ( $L m x)$ Fused and LmxULK4 in relation to UNC-51 like kinases. Maximum likelihood tree based on the $L G(+G+l)$ model of evolution and with 500 bootstrap replicates (MEGA 7 (Kumar et al., 2016); sequences used: H. sapiens ULK1 (O75385), ULK2 (Q8IYT8), ULK3 (Q6PHR2), ULK4 (Q96C45), STK36/Fused (Q9NRP7), PRKACA (P17612); M. musculus ULK1 (O70405), ULK2 (Q9QY01), ULK3 (Q3U3Q1), ULK4 (Q3V129), STK36/Fused (Q69ZM6), PRKACA (P05132); D. rerio ULK1 (F1R9T2), ULK2 (X1WEA3), ULK3 (A4IG43), ULK4 (A0A0R4IA69), Fused (A8WFS2), PRKACAA (A3KMS9); D. melanogaster ATG1/ULK1 (Q9VU14), ULK3 (Q9VHF6), Fused (P23647), PKA-C1 (P12370); A. thaliana RUK/ULK4 (F4JY37), with UniProt IDs in brackets (UniProt, 2021). T. brucei FCP5/ULK4 (Tb927.11.8150), FCP6/Fused (Tb927.11.4470); L. mexicana ULK4 (LmxM.28.0620), Fused (LmxM.13.0440) with TritrypDB GenelDs (Amos et al., 2021) in brackets. (B) Kinase motif ('G-loop', 'VAIK', 'HRD' and 'DFG' motifs) focused multiple sequence alignment. Catalytically relevant amino acid residues are highlighted in green, with the residues that form the canonical catalytic triad also highlighted in bold. (C-E) Fluorescence micrographs of $L$. mexicana promastigote forms, expressing either no fusion protein (parental control, C), LmxFused::mNG (D) or LmxULK4::mNG (E). The micrographs show different cell cycle stages, as assessed by the number of kinetoplasts $(K)$, nuclei $(N)$, and flagella (F). Nuclear and kinetoplast DNA were labelled with Hoechst 33342. Red arrows point to the base and tip of the flagellum.

Fig 2. Deletion of LmxULK4 and LmxFused results in loss of motile flagella.

(A) Swimming speed of the parental L. mexicana promastigote cells, LmxULK4 and LmxFused KO mutants and respective add-back cell lines. Each dot represents the average speed of promastigotes from one motility assay. (B) Proportion of cells with a visible external flagellum. (C) Measurement of flagellar length, for cells that had an external flagellum. Each dot represents an individual cell. (D) Morphology of the parental cell line, LmxULK4 and LmxFused KOs and episomal add-back cell lines. Images are composites of the phase contrast channel and a fluorescence channel showing nuclear and kinetoplast DNA labelled with Hoechst 33342. Arrows point to examples of cells with a visible external flagellum. An asterisk highlights example cells that lack a visible external flagellum.

Fig 3. Quantification of distinct flagellar morphotypes in LmxULK4 and LmxFused KO lines. (A) Cartoon depicting the expected flagellar localisation of fluorescently labelled RSP11::eYFP (green) and PF16::mStr (red) within the parental cell line. (B) Micrographs showing an example of each distinct flagellar morphotype observed in the tagged cell line (parental) and upon deletion of LmxFused (left panel) or LmxULK4 (right panel) ( $\mathrm{n} \geq 500$ ). Flagellar morphotypes were defined as follows: (i) a long external flagellum with no apparent defect (ii) a long flagellum with reduced or non-continuous PF16::mStr signal (highlighted by red arrows), (iii) a flagellum restricted to the flagellar pocket, (iv) a flagellum restricted to flagellar pocket lacking PF16::mStr signal, or (v) no flagellar structure detected, as assessed by the absence of fluorescently labelled proteins at the anterior cell pole where the flagellum normally emerges. (C) Cartoon depicting the expected flagellar localisation of PF16::eYFP 
1052 (green) and mStr::PF20 (red) within the parental cell line. (D) Micrographs showing an 1053 example of each distinct flagellar morphotype observed in the tagged cell line (parental) and 1054 upon deletion of $L m x F u s e d$ (left panel) or $L m x U L K 4$ (right panel) ( $\mathrm{n} \geq 500$ ). The composite 1055 images in ( $B$ and $D)$ are overlays of the phase contrast image with the red and green 1056 fluorescence channels and the Hoechst 33342 fluorescence indicating nuclear and 1057 kinetoplast DNA. (E-F) Comparison of flagellar PF16::eYFP and mStr::PF20 signal intensity 1058 in the parental cell line with $\Delta L m x F u s e d(E)$ and $\Delta L m x U l k 4$ (F) cell lines. This analysis only included cells with a long external flagellum with a continuous PF16::eYFP signal.

Fig 4. Ultrastructural defects in $\Delta$ LmxFused and $\Delta$ LmxUlk4 axonemes.

Transmission electron microscopy images showing cross-sections of flagellar axonemes. (A) Sections through the distal part of the flagellum outside of the flagellar pocket: (i) parental, (ii) $\Delta L m x U L K 4$, (iii, iv) $\Delta L m x F u s e d$. (B) Sections through the proximal part of the flagellum within the flagellar pocket show examples of normal $9+2$ microtubule arrangements in (i) parental and (ii) $\Delta L m x F u s e d$ flagella, and examples of ultrastructural defects in $\Delta L m x F u s e d$ (iii-vi). Colour labels indicate categories as quantified in C. (C) Quantification of ultrastructural defects of axonemes within the flagellar pocket. The categories were based on the number and arrangement of microtubules (MT). 9+2 (dark blue) represents the normal configuration of 9 radially symmetric outer doublet MTs and two singlet MT; ${ }^{*} 9+2$ indicates a collapse in the radial symmetry (light blue); $<9+2$ (yellow), $9+<2$ (dark pink), and $<9+<2$ (light pink) indicate deviations from the expected MT numbers.

1073

Fig 5. Acetylated $\alpha$-tubulin levels in $\Delta$ LmxULK4 and $\Delta$ LmxFused cell lines.

1075 (A) Acetylated a-tubulin was detected via immunofluorescence using monoclonal antibody C3B9 and TRITC-conjugated secondary antibody. DNA was labelled with Hoechst 33342. (B) Comparison of flagellar TRITC signal intensity between the parental control and $\triangle L m x U L K 4, \Delta L m x F u s e d$ and $\Delta L m x \alpha T A T 1$ deletion mutants ( $\mathrm{n} \geq 32$ cells). For $\Delta L m x U L K 4$ and $\Delta L m x F$ used, cells that displayed a long external flagellum were included in the analysis. (C) Percentage of cells displaying a visible long external flagellum that extends beyond the flagellar pocket in the parental cell line (99.8\%) populations of $\triangle L m x \alpha T A T 1(99.6 \%)$ $\triangle L m x U L K 4$ (5.4\%) and $\Delta L m x U L K 4 / \Delta L m x \alpha T A T 1$ (6.4\%) $(\mathrm{n} \geq 500)$.

1083

Fig 6. BiolD and IP data support an interaction between LmxFused and LmxULK4. (A) Proteins that were identified by BiolD only in the LmxFused::BirA* samples (B) Proteins identified by BiolD that were $\geq 5$ fold enriched within the LmxFused::BirA* samples relative to the parental control (Cas9T7M). (C) Proteins that co-immunoprecipitated with LmxFused::eYFP, and for which $\leq 1$ peptides were identified in the parental or GFP control sample. (D) Visualisation of LmxFused::mNG in live promastigotes before and after the deletion of $L m x U L K 4$. (E) Visualisation of LmxULK4::mNG in live promastigotes before and after deletion of LmxFused. The composite images show overlays of phase contrast images with the green fluorescence channels and fluorescence of Hoechst 33342 to indicate nuclear and kinetoplast DNA. (F) The percentage of cells displaying a visible long external flagellum that extends beyond the flagellar pocket was quantified in promastigote populations following combination with KO of LmxFused ( $n \geq 500)$. 
Fig 7. Localisation of ULK4 in mouse ependymal cilia.

1099 (A) Immunofluorescence staining of a multi-ciliated mouse ependymal cell. 3D reconstructed confocal z-stack using Imaris viewer (blend mode). Z-step size was $130 \mathrm{~nm}$. Acetylated tubulin (labeled with C3B9) in red, Flag-HA-mNeonGreen-tagged MmUlk4 (mNG fluorescence) in green, DNA (DAPI) in magenta. Note the strong $\mathrm{mNG}$ signal at tips of short axonemes and the weaker signal associated with tips of certain longer axonemes (arrows). Note that under used conditions the anti-acetylated antibody does not label centrioles in immunofluorescence. (B) Maximum intensity projection of confocal z-stack encompassing the apical region of two multi-ciliated cells, one expressing Flag-HA-mNeonGreen-tagged MmULK4 (bottom left) and the other not (top right). To improve the signal to noise ratio, we used $4 \mathrm{x}$ line averaging. Raw data are presented. Left- ciliary marker Arl13b (labeled with anti-Arl13b antibody) in red, Flag-HA-mNeonGreen-tagged MmUlk4 (mNG fluorescence) in green. Right- mNG signal only. (C) Selected confocal planes of an apical region of an expanded multi-ciliated mouse ependymal cell, which encompasses centrioles. Individual planes are separated by $700 \mathrm{~nm}$ in the axial direction. Individual centrioles (labeled with C3B9; in red) are denoted with numbers. Note the Flag-HA-mNeonGreen-tagged MmUlk4 signal (anti-HA staining; in green) capping one end of each centriole. (D) 3D reconstruction of a confocal z-stack encompassing distal parts of cilia of an expanded multi-ciliated mouse ependymal cell. Z-step size was $100 \mathrm{~nm}$ and Imaris viewer (blend mode) was used for the reconstruction. Acetylated tubulin (labeled with C3B9) in red, Flag-HA-mNeonGreen-tagged MmULK4 (anti-HA signal) in green. Arrows indicate the tips of axonemes with detectable 1119 MmULK4.

Fig 8. Models for possible interactions between ULK4 and Fused/STK36 autophosphorylation of the LmxFused activation loop to contribute to flagellum assembly or microtubule stabilisation. In Model B, LmxFused forms a homodimer. In this context it is not yet clear if LmxULK4 interacts with LmxFused to promote; (i) assembly of the regulatory spine (R-spine), which is a key feature of activated kinases, (ii) homodimerization, (iii) activation loop phosphorylation and/or (iv) the interaction of LmxFused with downstream targets. Stacked red circles represent the formation of the R-spine. Yellow circles represent 
bioRxiv preprinks doi: https://doi.org/10.110/1/2022:03.06.483169; this version posted March

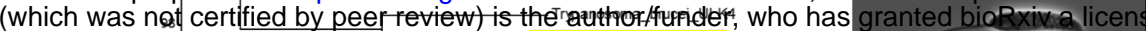

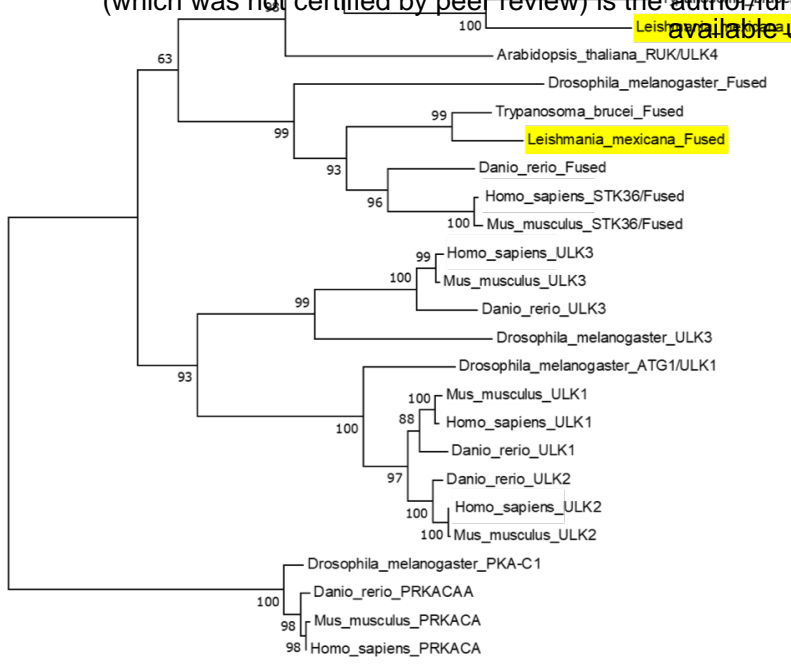

\section{B}

H. sapiens $\mathrm{PKA}$

H. sapiens ULK 1

H. sapiens ULK 2

H. sapiens ULK 3

D. melanogaster Fused

D. rerio STK36/Fused

M. musculus STK36/Fused

H. sapiens STK36/Fused

T. brucei Fused

L. mexicana Fused

A. thaliana RUK/ULK4

M. musculus ULK4

H. sapiens ULK4

T. brucei ULK4

L. mexicana ULKA

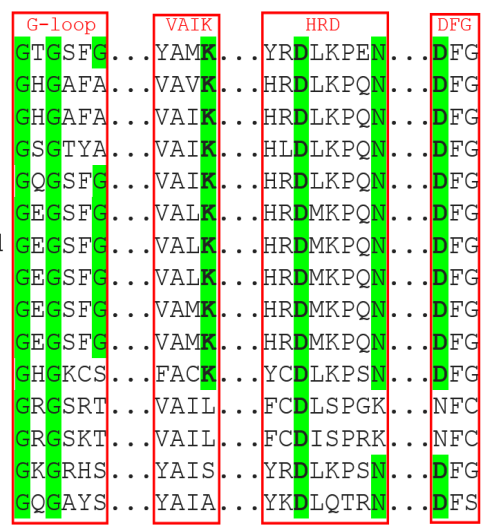

D

5um

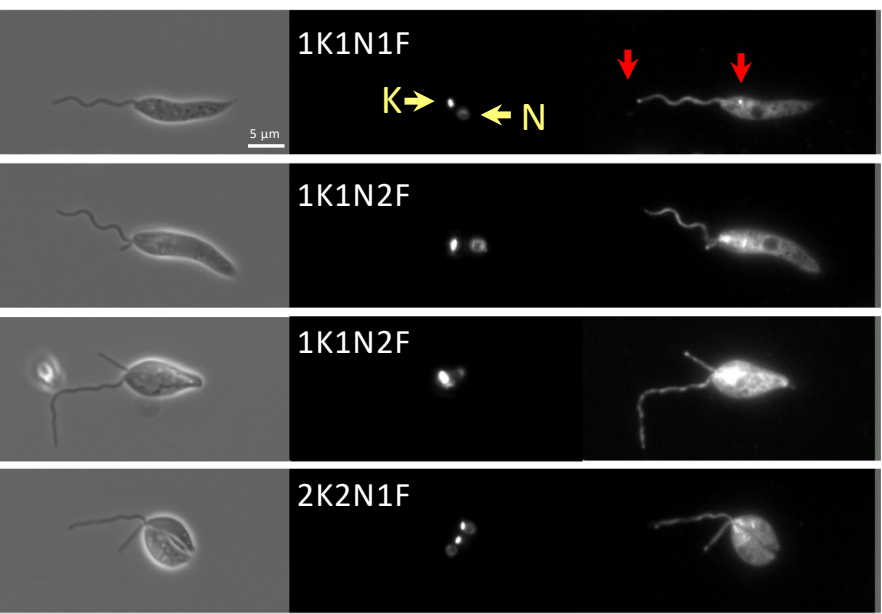

E
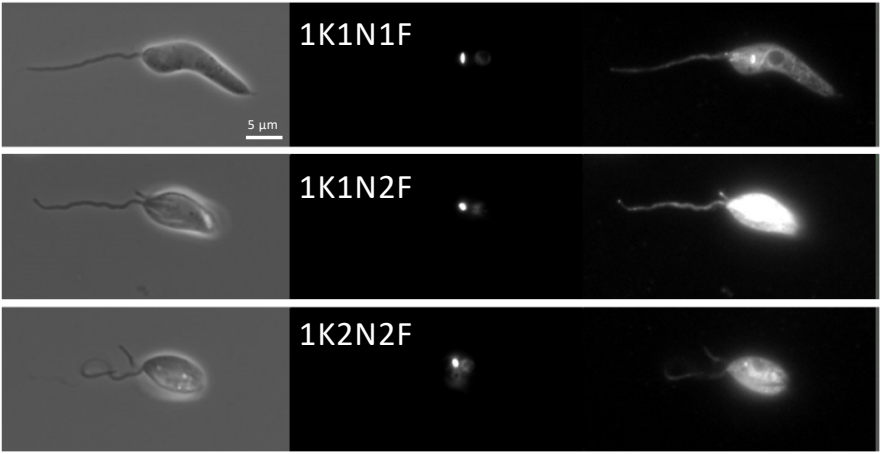

2K2N1F

;

\section{Figure 1}




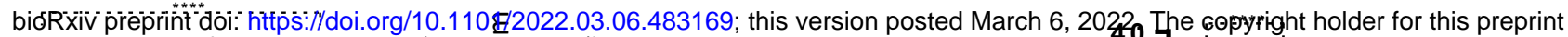

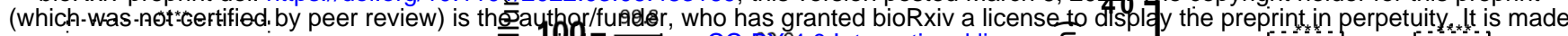
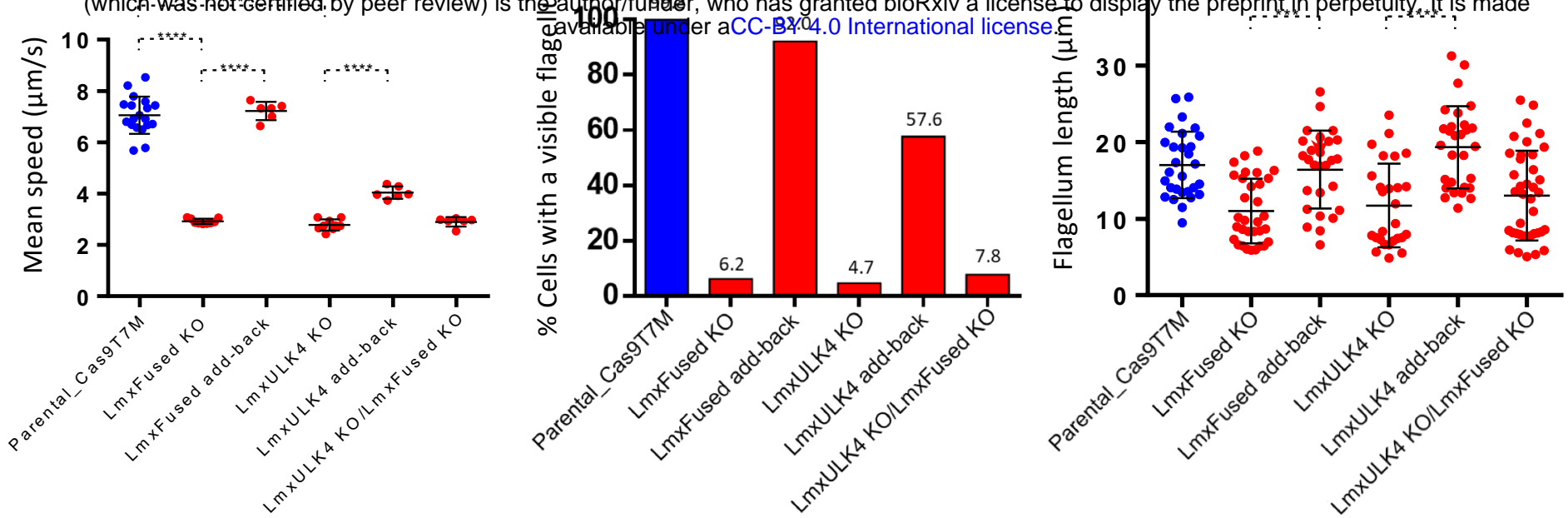

D
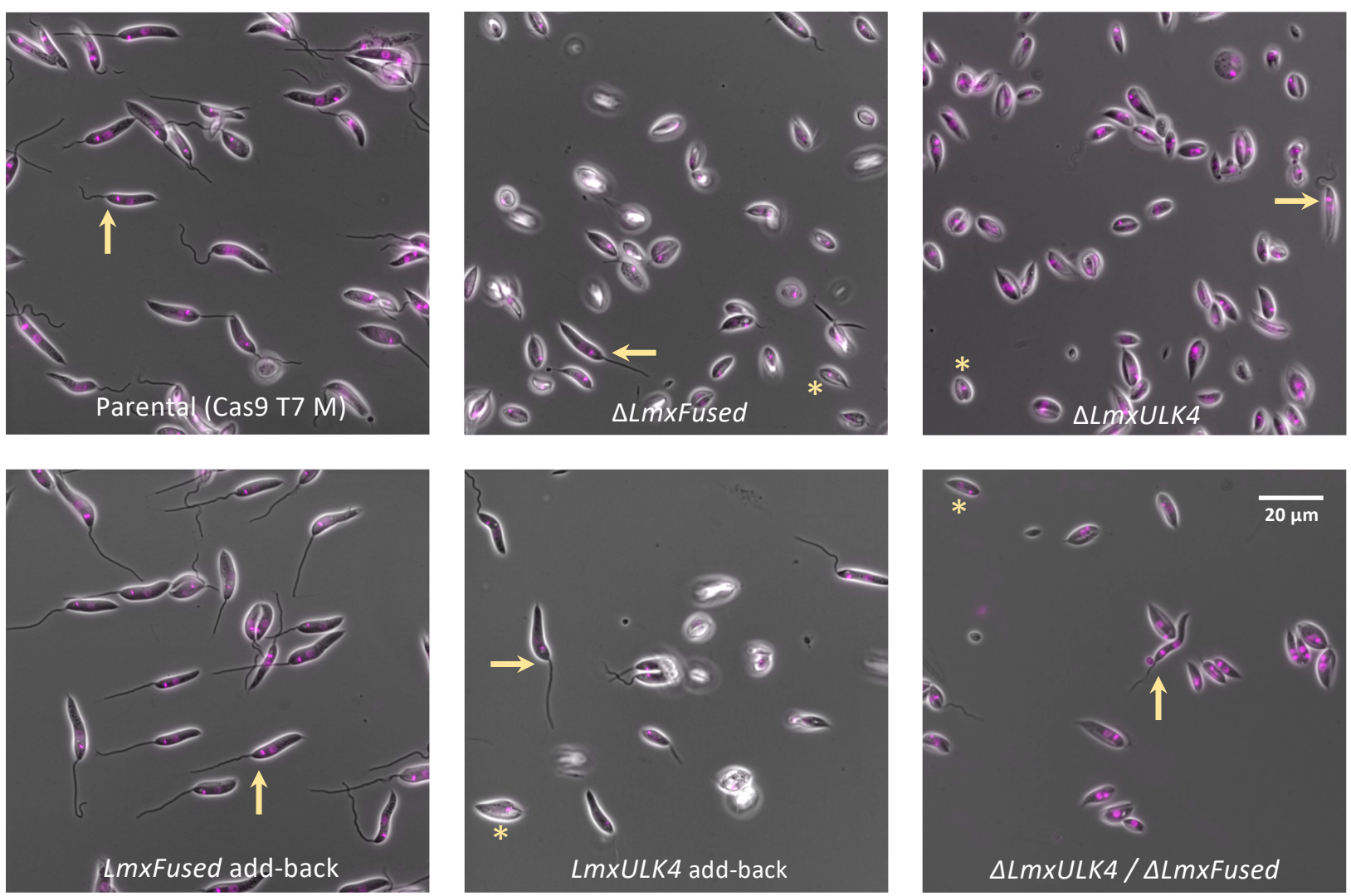

Figure 2 
A

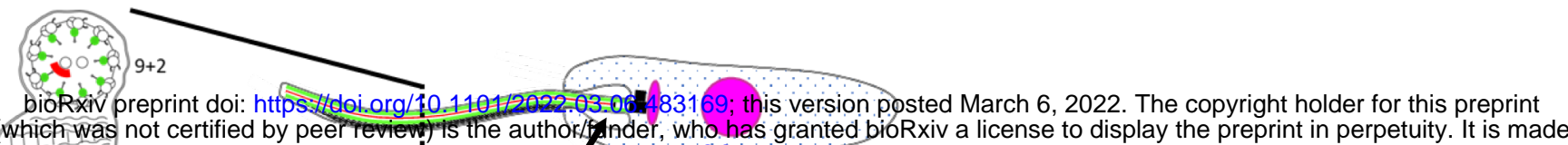
$=$ PFR available under aCG-BY 4.O International license.

B Flagellar pocket eYFP mStrawberry Composite
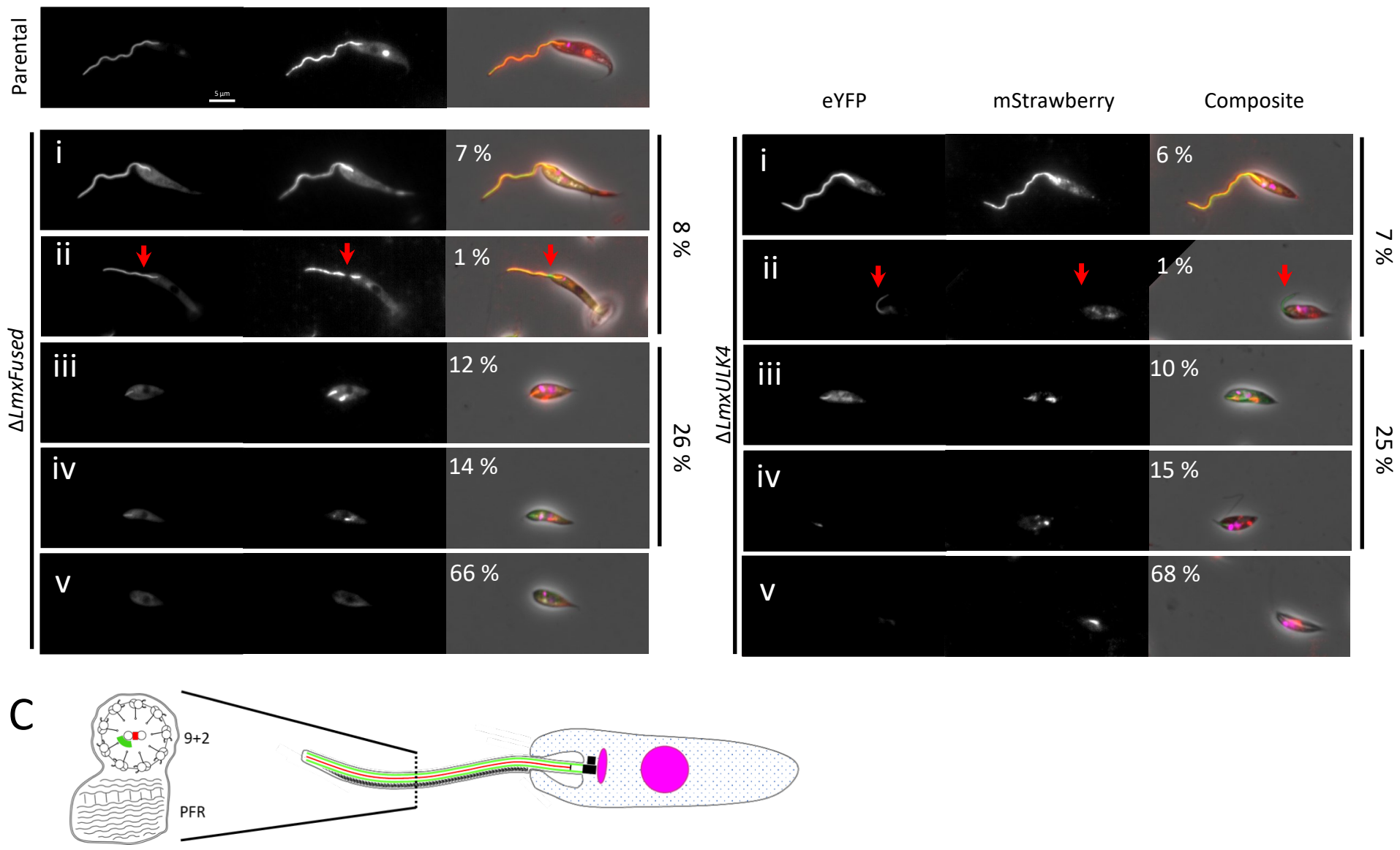

D
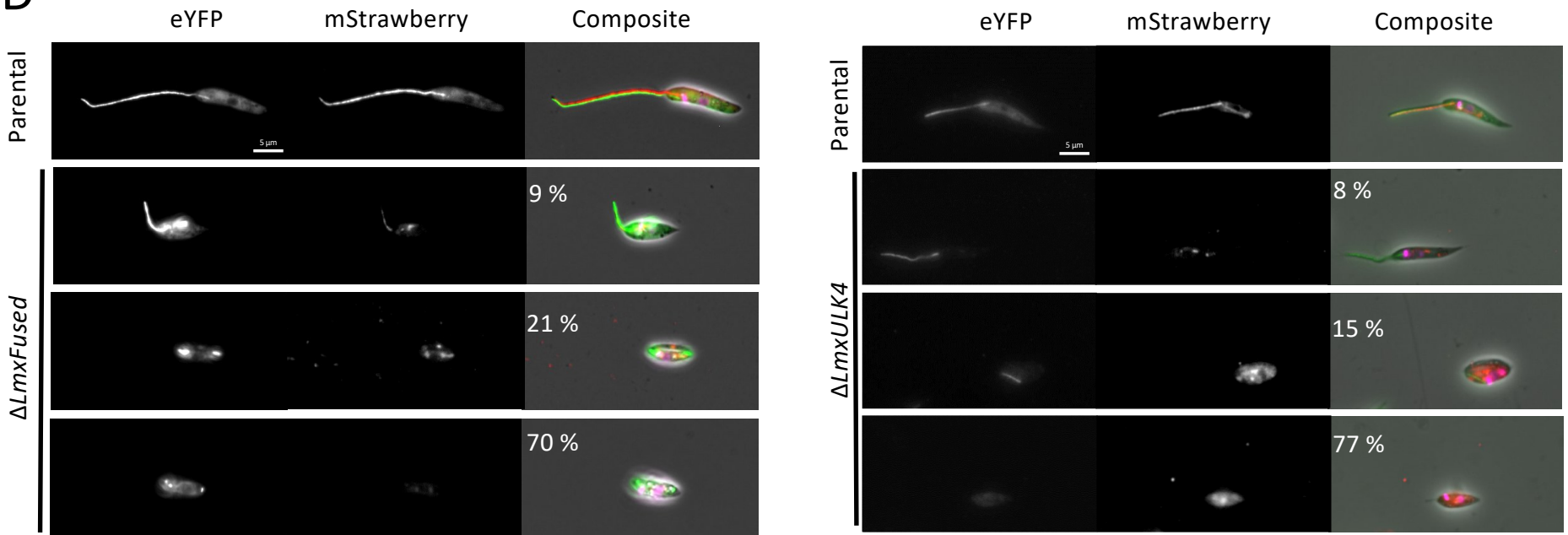

$\mathrm{E}$

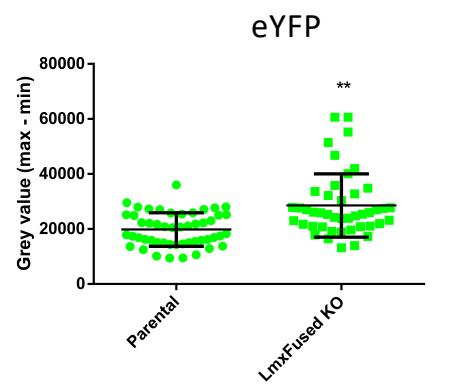

mStrawberry

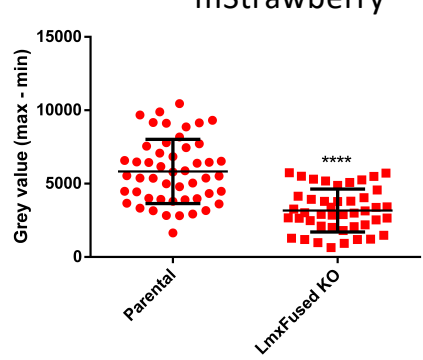

$\mathrm{F}$

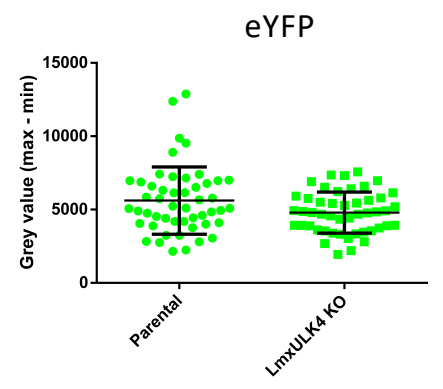

Figure 3 
bioRxiv preprint doi: https://doi.org/10.1101/2022.03.06.483169; this version posted March 6, 2022. The copyright holder for this preprint (which was not certified by peer review) is the author/funder, who has granted bioRxiv a license to display the preprint in perpetuity. It is made available under aCC-BY 4.0 International license.

A

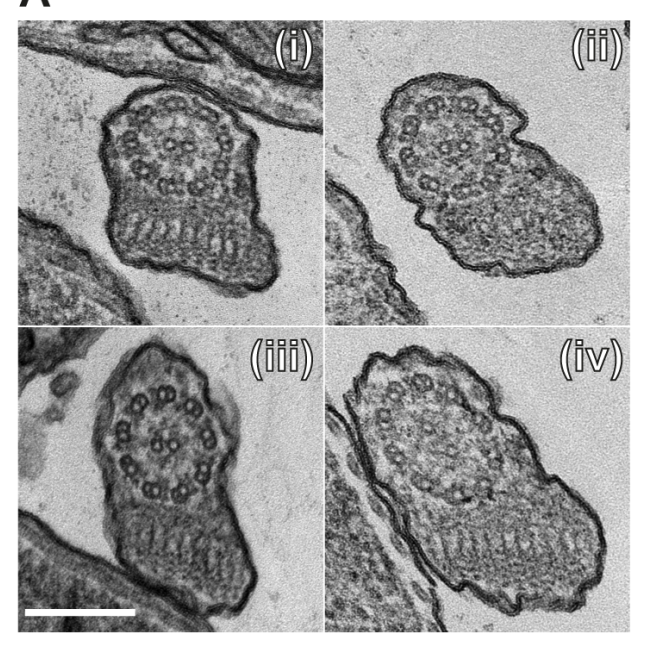

B

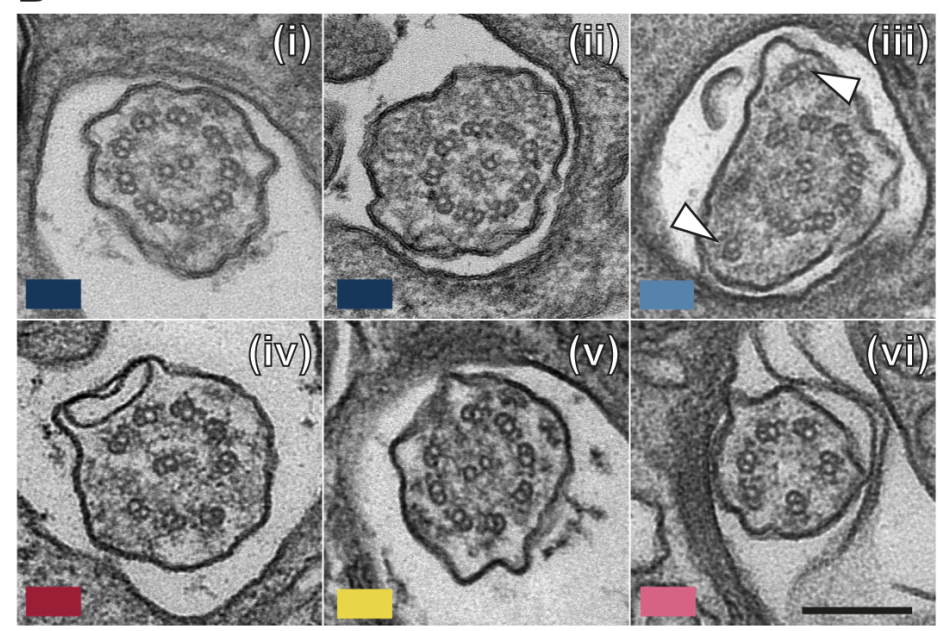

$50 \%$

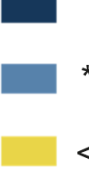

$9+2$

$* 9+2$

$<9+2$

\section{Figure 4}




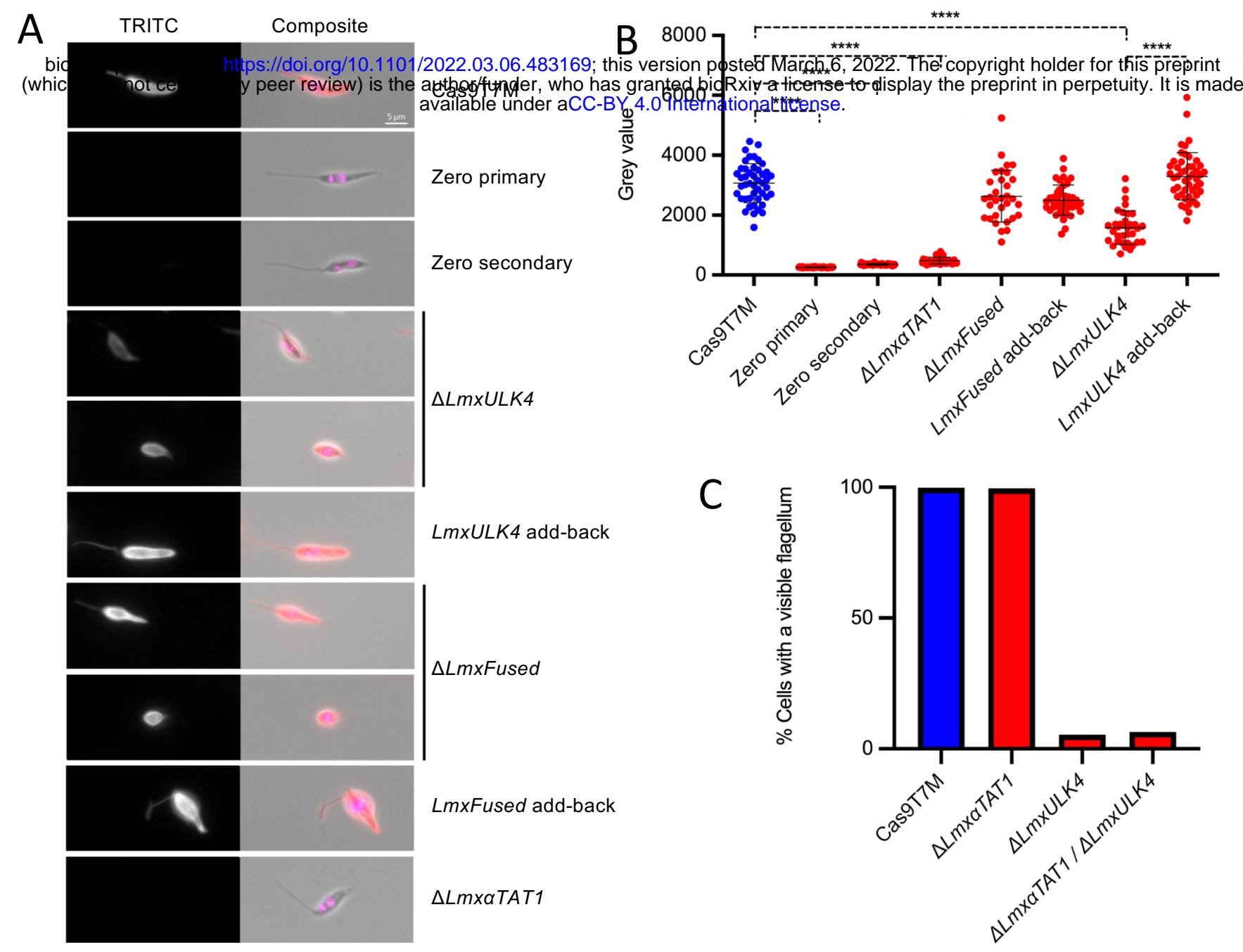

\section{Figure 5}


A 50

B

14

C 1000

45bioRxiv preprint doi: https://doi.org/10.1101/2022.03.06.483169; this version posted March 6, 2022. The copyright holder for this preprint
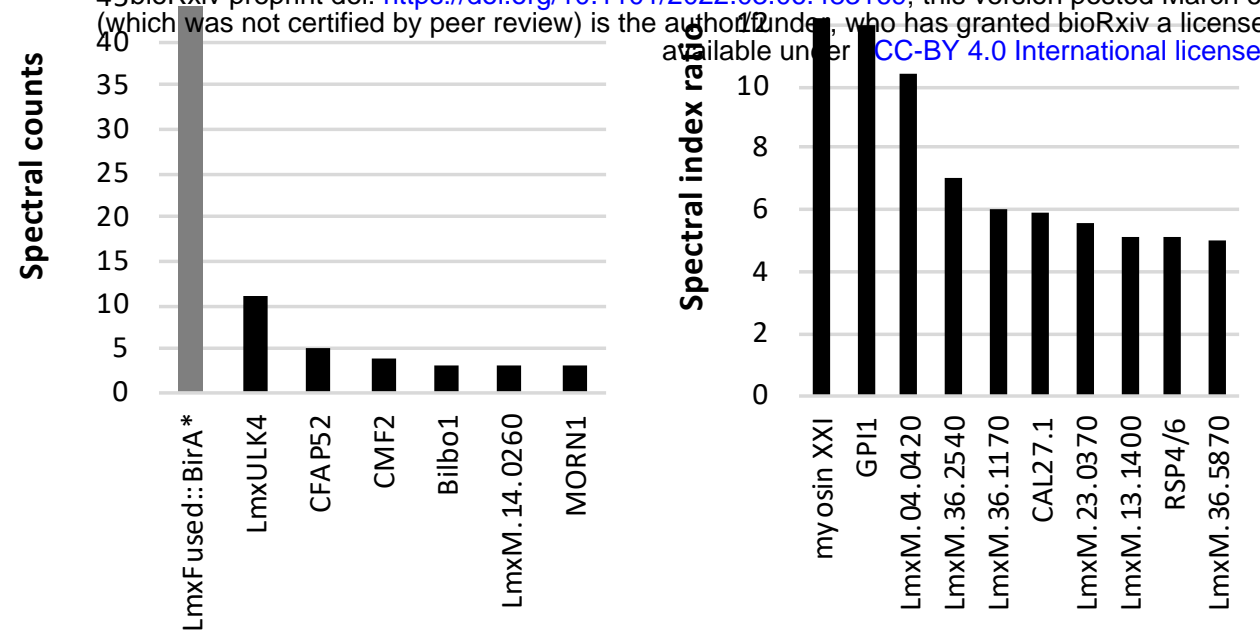

display the preprint in perpetuity. It is made

\section{D}

mNeonGreen
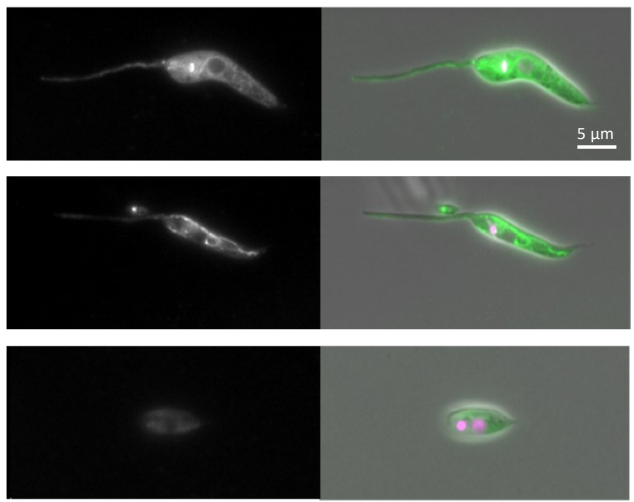

LmxFused::mNG

/ $\triangle L m x U L K 4$

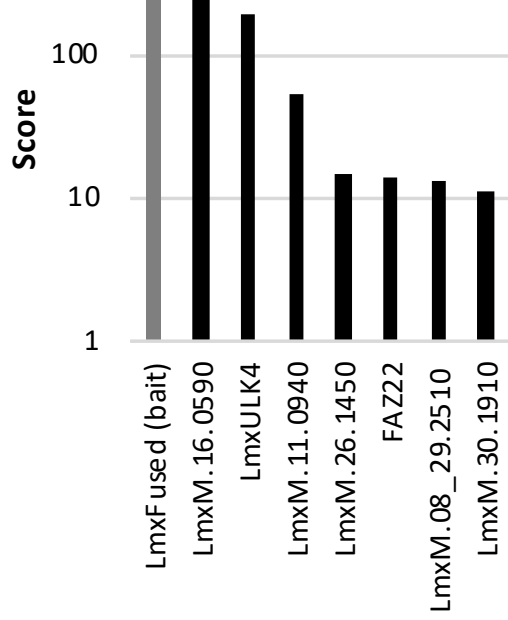

E
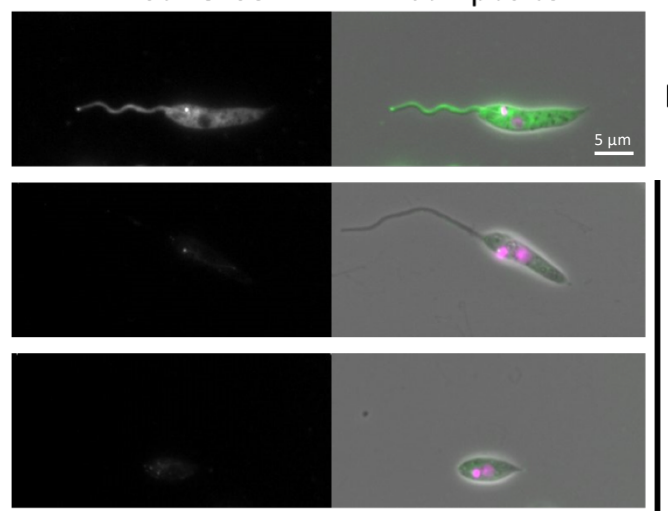

LmxULK4::mNG /DLmxFused

$\mathrm{F}$

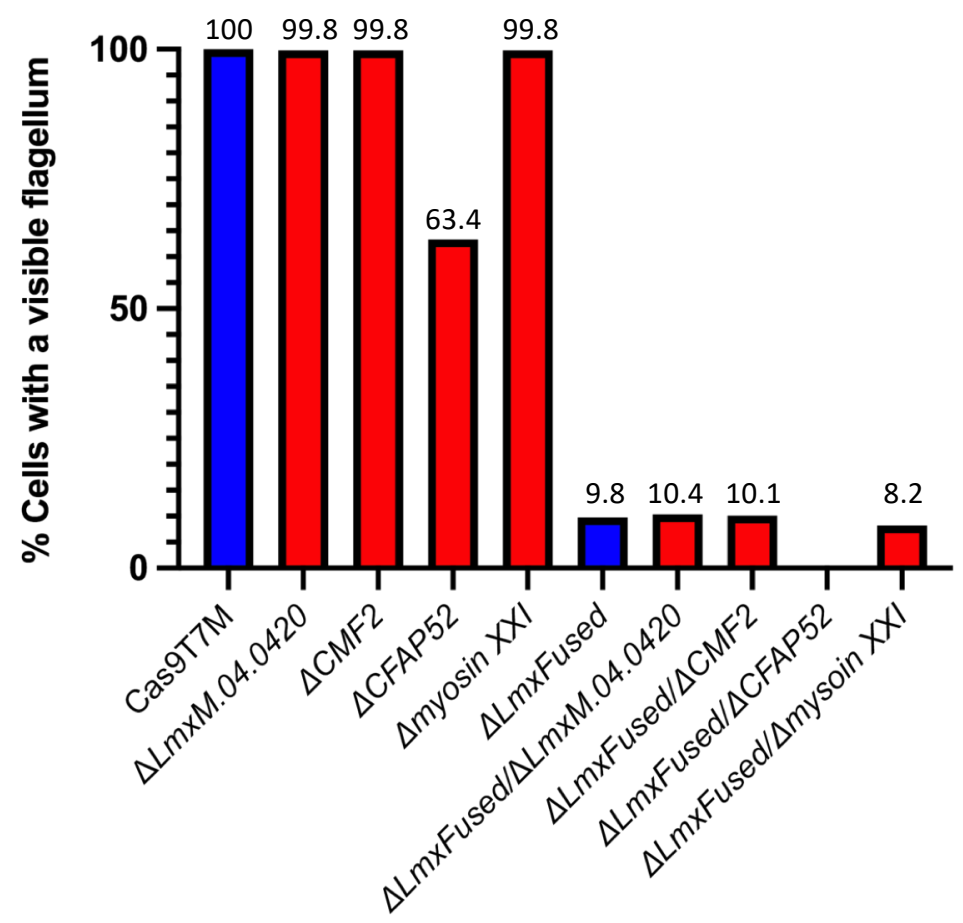

Figure 6 
biofAiv preprint doi: https://doi.org/10.1101/2022.03.06.483169; this @ersion posted March 6, 2022. The copyright holder for this preprint

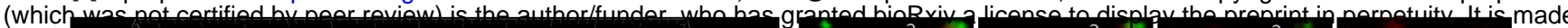
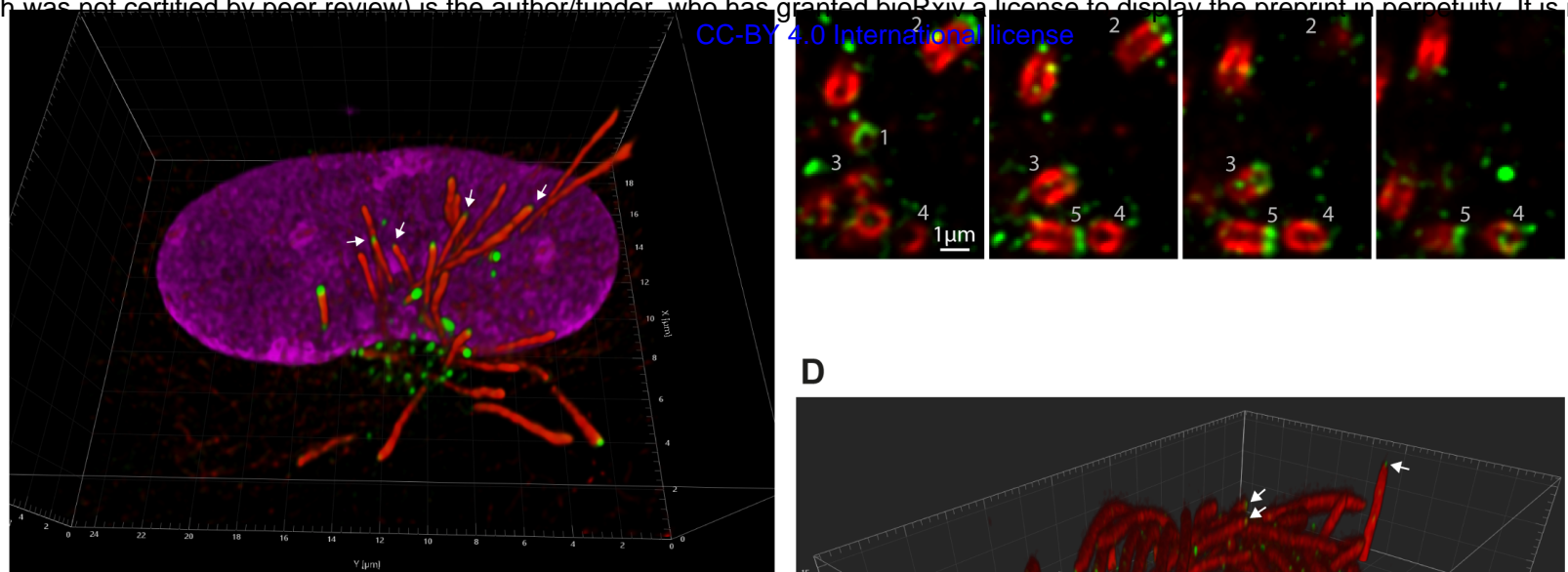

B
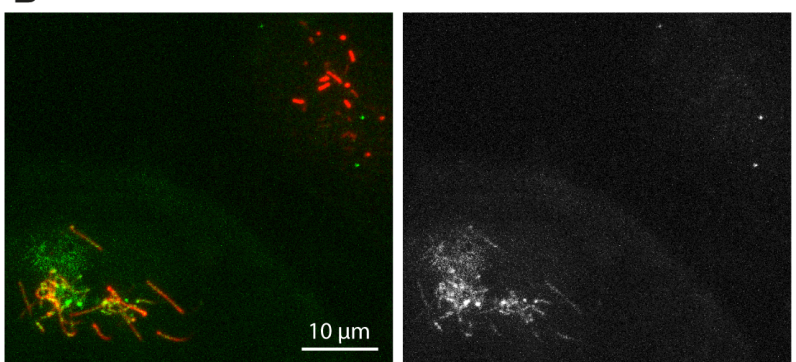

D

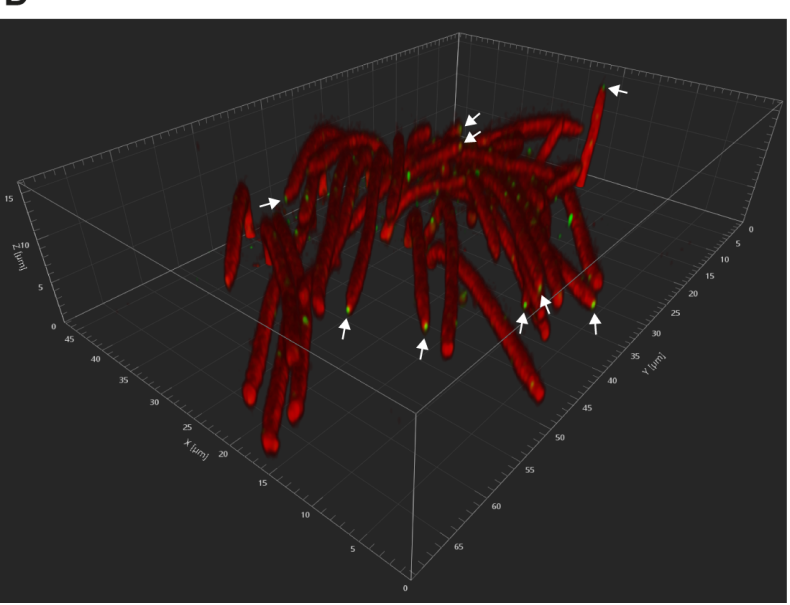

\section{Figure 7}




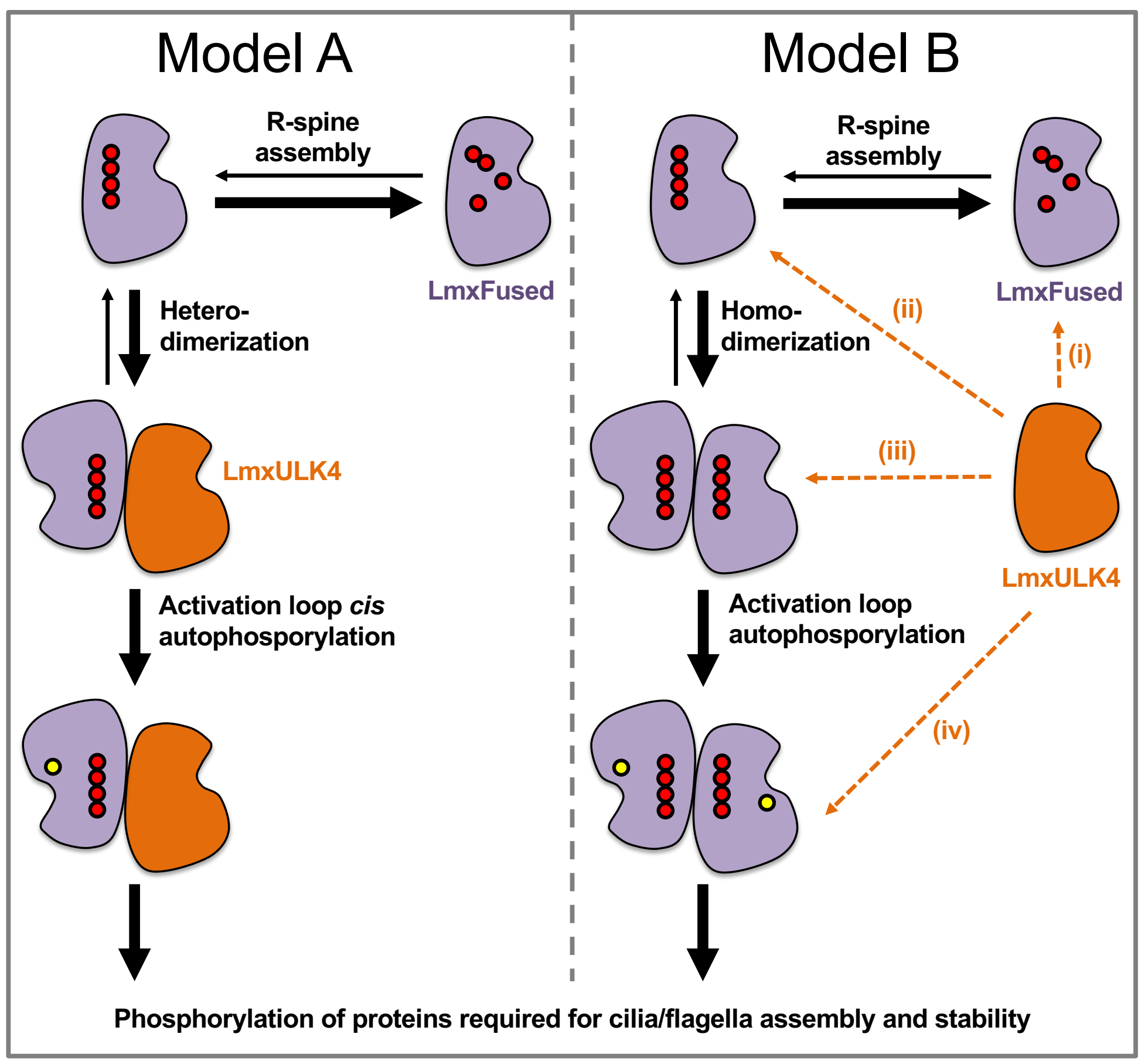

Figure 8 